\title{
The N6-methyladenosine-modified Pseudogene HSPA7 Correlates with the Tumor Microenvironment and Predicts the Response to Immune Checkpoint Therapy in Glioblastoma
}

\section{Rongrong Zhao}

Shandong University Qilu Hospital

\section{Boyan Li}

Shandong University Qilu Hospital Department of Cardiology

Shouji Zhang

Shandong University Qilu Hospital

Zheng He

Shandong University Qilu Hospital

\section{Ziwen Pan}

Shandong University Qilu Hospital

\section{Xu Li}

Shandong University Qilu Hospital

\section{Qindong Guo}

Shandong University Qilu Hospital

\section{Wei Qiu}

Shandong University Qilu Hospital

\section{Yanhua Qi}

Shandong University Qilu Hospital

\section{Shulin Zhao}

shandong university Qilu hospital

\section{Shaobo Wang}

shandong university Qilu hospital

\section{Zihang Chen}

Shandong University Qilu Hospital

\section{Ping Zhang}

shandong university qilu hospital

\section{Xing Guo}

Shandong University Qilu Hospital

\section{Hao Xue}

shandong university qilu hospital 
Gang Li ( $\square$ dr.ligang@sdu.edu.cn )

Shandong University Qilu Hospital

\section{Research}

Keywords: N6-methyladenosine, long noncoding RNA, Tumor microenvironment, Glioblastoma, Immune checkpoint blockade

Posted Date: December 22nd, 2020

DOl: https://doi.org/10.21203/rs.3.rs-130869/v1

License: (c) (i) This work is licensed under a Creative Commons Attribution 4.0 International License. Read Full License 


\section{Abstract}

Background: Glioblastoma (GBM), one of the most aggressive cancers in brain tumor type, have no effective and sufficient therapies. Identifying robust biomarkers of immune checkpoint blockade (ICB) therapy, a promising treatment option for GBM patients, are urgently warranted.

Methods: We comprehensively evaluated the $\mathrm{m}^{6} \mathrm{~A}$ modification patterns of IncRNAs via $\mathrm{m}^{6} \mathrm{~A}$-sequencing $\left(\mathrm{m}^{6} \mathrm{~A}-\mathrm{seq}\right)$ data of GBM tissues. And systematically investigated the immune and stromal regulators in these $\mathrm{m}^{6} \mathrm{~A}$ regulated IncRNAs. We used the ssGSEA (single-sample gene-set enrichment analysis) algorithm to investigate the difference on enrichment of TME cell infiltration and functional annotation of HSPA7 in individual GBM sample.

Results: We depicted a transcriptome-wide $\mathrm{m}^{6} \mathrm{~A}$ methylation profiling of IncRNAs in GBM for the first time, revealing the highly distinct $\mathrm{m}^{6} \mathrm{~A}$ modification patterns of IncRNAs, compared to the normal brain tissues. And we identified $\mathrm{m}^{6} \mathrm{~A}$ modified pseudogene HSPA7 as a novel risk prognostic factor for GBM patients, playing crucial roles in immunophenotype, stromal and carcinogenic pathways activation, having a robust predictive capacity of ICB immunotherapeutic response.

Conclusions: This work revealed that the $\mathrm{m}^{6} \mathrm{~A}$ modified IncRNA HSAP7 played a nonnegligible role in tumor microenvironment (TME) cell infiltration, stromal and carcinogenic activation, and could be a robust predictive biomarker of ICB immunotherapy for GBM patients.

\section{Background}

Glioblastoma (GBM), as one of the most aggressive cancers in brain tumor type, have no effective and sufficient therapies due to its intra-tumoral heterogeneity and molecular complexity so far. Immune checkpoint blockade (ICB) therapy is being actively pursued for GBM as a promising treatment option. However, very few patients respond it ${ }^{[1-5]}$, partly result from the attribution of prominent immunesuppressive players of the brain tumor microenvironment (TME) in GBM, including tumor-associated macrophages (TAMs), neutrophils and Tregs ${ }^{[6-8]}$. Developing biomarkers and uncovering the concrete resistant molecular mechanism of ICB therapy is in urgent need to identify effective therapeutic strategies for GBM.

$\mathrm{N}^{6}$-Methyladenosine $\left(\mathrm{m}^{6} \mathrm{~A}\right)$, the most abundant reversible methylation of mRNA, has a critical effect on mRNA metabolism, including splicing, export, translation and decay. And dysregulation of this modification is clearly linked to the diverse physiological processes and disease progression $[9,10]$, including GBM tumorigenesis ${ }^{[1-15]}$. Recent studies described the role of the $\mathrm{m}^{6} \mathrm{~A}$ modification in regulating the immune response ${ }^{[16-19]}$, prompting us the importance in portraying $\mathrm{m}^{6} \mathrm{~A}$ regulated gene spectrum and $\mathrm{m}^{6} \mathrm{~A}$ regulatory mechanisms in shaping the TME. It has been demonstrated by numerous researches that $\mathrm{m}^{6} \mathrm{~A}$ has also been discovered in numberous long noncoding RNAs ${ }^{[15,20-22]}$ (IncRNAs, whose transcripts were more than 200 nucleotides in length, but lack functional coding capacity), 
emerged as fundamental players in cancer progression and immune regulation, suggesting a potential association between cancer immune response and $\mathrm{m}^{6} \mathrm{~A}$ IncRNA modification. However, the $\mathrm{m}^{6} \mathrm{~A}$ methylation profiling of IncRNAs have not been systematically clarified in GBM tumors. Additionally, none of these reports have specifically investigated the roles of $\mathrm{m}^{6} \mathrm{~A}$ modified IncRNAs in the overall TME landscape of the GBM. Therefore, it is worthy to get a comprehensive knowledge in the TME cell infiltration characterizations mediated by $\mathrm{m}^{6} \mathrm{~A}$ modified IncRNAs, contributing to our understanding of the $\mathrm{m}^{6} \mathrm{~A}$ modification in the immune regulation and guiding more effective immunotherapy strategies.

Here, we depicted a transcriptome-wide $\mathrm{m}^{6} \mathrm{~A}$ methylation profiling of IncRNA in GBM and the normal brain tissues via $\mathrm{m}^{6} \mathrm{~A}$-seq data for the first time, revealing the highly distinct $\mathrm{m}^{6} \mathrm{~A}$ modification patterns of IncRNAs between these two groups. Key immune-stromal-related IncRNAs were identified by the differentially expressed gene (DEG) analysis in TCGA primary GBM cohorts. Integrating our own revealed $\mathrm{m}^{6} \mathrm{~A}$ regulated IncRNAs, we identified HSPA7 as a novel risk prognostic factor for GBM patients. Through detailed bioinformatics analyses of HSPA7, we found its crucial role in immunophenotype determination, stromal activation and carcinogenic pathways activation, and highlighted its robust predictive capacity of ICB immunotherapeutic response.

\section{Methods}

\section{Patients specimens and public patient cohorts}

Human GBM and normal brain tissues for $\mathrm{m}^{6} \mathrm{~A}$-sequencing $\left(\mathrm{m}^{6} \mathrm{~A}\right.$-seq), were obtained from patients admitted to Qilu Hospital. All participants provided written informed consent, and the research was approved by the Ethical Committee on Scientific Research of Shandong University Qilu Hospital (approval number: KYLL-2018-324).

The TCGA GBM RNA-seq transcriptome, somatic mutation data and corresponding clinicopathological parameters were obtained from the TCGA database (http://cancergenome.nih.gov/); Two CGGA GBM RNA-seq data and corresponding clinicopathological parameters were obtained from CGGA database (http://www.cgga.org.cn/). For immune-checkpoint blockade genomic and clinical information in IMvigor210 cohort, based on the Creative Commons 3.0 License, the complete expression data and detailed clinical annotations were obtained from http://research-pub.Gene.com/imvigor210corebiologies. For data of GBM patients, treated with PD-1 inhibitors (pembrolizumab or nivolumab), and clinical information were obtain from supplementary data of paper and the sequencing data were obtain from SRA PRJNA482620 ${ }^{[2]}$. For patients with melanoma treated with anti-CTLA4 therapy, the expression data were downloaded from cBioPortal database (http://www.cbioportal.org/), and the detailed clinical characteristics of individual patients get from the supplementary data of paper ${ }^{[23]}$. And the Fig.S11A somatic mutation data and information were obtained from cBioPortal database. The $\mathrm{m}^{6} \mathrm{~A}$-seq sequencing data have been deposited in SRA PRJNA661159 (the data is being processed, submission ID: 
SUB8069560, released when the paper is published). And the processed data is available from the corresponding author on reasonable request.

\section{Estimation of TME cell characterization}

We used the SSGSEA (single-sample gene-set enrichment analysis) algorithm to quantify the enrichment score of each sample immune cell infiltration in the GBM TME. The genes for immune cell types were obtained from Bindea et al. (Table S1) ${ }^{[24]}$, including immune cell types, immune-related pathways and functions. And based on the results of SsGSEA, samples of TCGA GBM cohort were classified as high immune cell infiltration group (immune-H) and low immune cell infiltration group (immune-L) by using "hclust" R package.

\section{Functional annotation and enriched pathways analysis}

To explore the biological behaviors among these distinct HSPA7 expression samples, we used some gene sets of HALLMARK ${ }^{[25]}, \mathrm{KEGG}^{[26]}$ from MSigDB database and some other prevalent gene signature ${ }^{[27-29]}$ to estimate pathway enrichment scores for each sample (Table S2), for GSVA enrichment analysis using "GSVA" R packages.

The enriched terms of genes, positively correlated with the expression of HSPA7, and genes interacting with HSPA7 detected in NCBI (https://www.ncbi.nlm.nih.gov/gene/3311) and starbase database (http://starbase.sysu.edu.cn/index.php), were analyzed via matoscape tool (http://metascape.org/gp/index.html\#/).

\section{Statistical analysis}

Kaplan-Meier survival curves were performed using GraphPad Prism 7.04, and significant differences were compared by the log-rank (Mantel-Cox) test between two groups. The waterfall function of "maftools" package was performed to visual the mutation landscape in patients with high and low HSPA7 group or high and low TMB group. Student's t-test was used for two group comparison. For comparisons of more than two groups, Kruskal-Wallis or one-way ANOVA tests were used for nonparametric and parametric methods ${ }^{[30]}$. $P \leq 0.05$ was considered statistically significant $\left({ }^{\star} P \leq 0.05 ; * \star P\right.$ $\leq 0.01 ; * \star * P \leq 0.001, * \star \star * P \leq 0.0001)$. All data processing with $\mathrm{R}$ packages were performed using $\mathrm{R}$ Studio (version 3.6.3)

\section{Results}

\section{Overview of $\mathrm{m}^{6} \mathrm{~A}$ transcriptome methylation within IncRNAs in GBM and normal brain tissues}


To understand the pattern of the $\mathrm{m}^{6} \mathrm{~A}$ modification profiles of IncRNAs in GBM, 3 human GBM tumor tissues versus 3 normal brain tissues were conducted for transcriptome $\mathrm{m}^{6} \mathrm{~A}-\mathrm{seq}$, which revealed that a considerable fraction of IncRNAs were extensively $\mathrm{m}^{6} \mathrm{~A}$-modified. And, a total of $2113 \mathrm{~m}^{6} \mathrm{~A}$ peaks were identified in the normal group, representing transcripts of 1538 genes, including 544 lincRNAs (intergenic IncRNAs), 720 antisense IncRNAs and 262 pseudogenes and 12 others; While, in GBM group, $2412 \mathrm{~m}^{6} \mathrm{~A}$ peaks were identified, corresponding with the transcripts of 1508 genes, consist of 445 lincRNAs, 771 antisense IncRNAs and 283 pseudogenes and 9 others (Fig. 1A). Further analysis found that the majority of IncRNAs (74.5\% of the $\mathrm{m}^{6} \mathrm{~A}$-methylated genes in the normal group, while $65.3 \%$ of the methylated genes in the GBM group) contained only one peak in both groups, while a relatively small number of genes contained two peaks, and very few IncRNAs contained three or more peaks (Fig. 1B). To further analyze the distribution profiles of $\mathrm{m}^{6} \mathrm{~A}$ peaks within IncRNAs, we found that $\mathrm{m}^{6} \mathrm{~A}$ site is distributed in intron and exon regions in the almost the same proportion in normal group; While, A little bit more inclined to distribute in exon regions in GBM tissues, compared to normal group (Fig. 1C).

To uncover the significance of $\mathrm{m}^{6} \mathrm{~A}$ methylated IncRNAs in GBM, the differences and overlaps in $\mathrm{m}^{6} \mathrm{~A}$ modified IncRNAs between the individuals were analyzed by the Venn diagram. As shown in Fig. 1D, $781 \mathrm{~m}^{6} \mathrm{~A}$-modified IncRNAs were detected within both groups. Compared with the normal group, the GBM group had 727 new genes, with disappearance of 757 genes, indicating a significant difference in global IncRNA $\mathrm{m}^{6} \mathrm{~A}$ modification patterns between the GBM and normal brain groups. To explore the effect of $\mathrm{m}^{6} \mathrm{~A}$ on IncRNA expression, the differentially expressed IncRNAs between 153 primary GBM and 5 normal brain tissues in TCGA GBM cohort were compared. Compared with normal samples, 4375 IncRNAs were differentially expressed in GBM tissues ( $\operatorname{LogFC} \geq 1$ and padj $\leq 0.05$ ), with 2614 upregulated genes and 1761 downregulated genes (Table S3). And, the global abundance of the $\mathrm{m}^{6} \mathrm{~A}$ peaks between GBM and normal brain tissues were also compared. Further, conjoint analysis of $\mathrm{m}^{6} \mathrm{~A}$ diff-modified IncRNAs and TCGA diff-regulated IncRNAs were conducted, and the distribution of genes with a significant change in both $\mathrm{m}^{6} \mathrm{~A}$ level $(|\mathrm{FC}| \geq 1.2, \mathrm{p} \leq 0.05)$ and overall transcript expression level $(|\mathrm{FC}| \geq 2$, padj $\leq 0.05)$ were shown in Fig. 1E, which were mainly divided into four groups, including 208 hypermethylated as well as upregulated genes ('hyper-up'), 394 hypomethylated as well as downregulated genes ('hypo-down'), 56 hypermethylated but downregulated genes ('hyper-down') and 64 hypomethylated but upregulated genes ('hypo-up') in GBM tissues relative to normal brain tissues. And we discovered a positive correlation of differentially methylated $\mathrm{m}^{6} \mathrm{~A}$ peaks and their corresponding gene expression levels (Fig. 1E, pearson correlation $r=0.495, p<0.0001$ ), and the $m^{6} \mathrm{~A}$ modification sites of the above four group genes were all distributed in the exon region, reconfirming that $\mathrm{m}^{6} \mathrm{~A}$ modification can regulate the expression of mature IncRNAs. These results showed that there was an obviously distinct $\mathrm{m}^{6} \mathrm{~A}$ modification patterns between GBM and normal brain tissues. And $\mathrm{m}^{6} \mathrm{~A}$ modification could regulate a large amount IncRNAs, may via regulating their stability, degradation or some other functions, having been reported previously. 


\section{Identification of immune-stromal-m ${ }^{6} \mathrm{~A}-$-related pseudogene HSPA7 a novel risk prognostic factor in GBM}

To investigate the effects of these $\mathrm{m}^{6} \mathrm{~A}$ regulated IncRNAs on TME cell infiltration, we assessed tumorpurity, stromal and immune scores of 153 primary GBM cases in TCGA GBM cohort, using the ESTIMATE algorithm (see Methods, Table S4). Then we performed differential analysis of all RNA-seq data from 153 GBM samples in TCGA database, based on the median cutoff immune/stromal scores, respectively. And the volcano plot of the high/low scores of the stromal/immune scores revealed differential gene expression profiles between the samples, in which 791 up-regulated IncRNAs and 459 down-regulated IncRNAs $(|\mathrm{FC}| \geq 1.5$, padj $\leq 0.05)$ were obtained based on the difference in immune scores (Fig. S1A, Table S5), simultaneously, 734 up-regulated IncRNAs and 269 down-regulated IncRNAs $(|F C| \geq 1.5$, padj $\leq 0.05)$ were obtained based on the differential analysis of stromal scores (Fig. S1B, Table S6), As can be seen from the venn diagram (Fig. S1C-D), there were 4 identical up-regulated genes and 11 identical down-regulated genes, related to immune, stromal and $\mathrm{m}^{6} \mathrm{~A}$. Then, we performed Kaplan-Meier curve analysis on the obtained 15 differential genes, only HSPA7 (Fig. 2B) and AC011899.9 (Fig. S2B) had prognostic significance $(p \leq 0.05)$ in 153 TCGA GBM samples based on the median expression. The GEPIA database showed that HSPA7 was significantly overexpressed in GBM tissues, compared with GETx normal brain tissues (Fig. 2A), while AC011899.9 was not (Fig.S2A). Thus, we focused only on HSPA7 in the following work. And the $\mathrm{m}^{6} \mathrm{~A}$ methylation peak distribution and abundances in HSPA7 transcript of GBM and normal brain tissues, detected by $\mathrm{m}^{6} \mathrm{~A}-\mathrm{seq}$, was visualized using IGV software (Fig. 2C). And We found that HSPA7 was highly enriched in the $\mathrm{m}^{6} \mathrm{~A}$ precipitated fraction, and the $\mathrm{m}^{6} \mathrm{~A}$ modification enrichment level could be regulated by methyltransferase like 3 (METTL3), containing catalytic activity domain to catalyze the $\mathrm{m}^{6} \mathrm{~A}$ formation ${ }^{[31]}$ (Fig. 2D). Also, the expression of HSPA7 could be significantly inhibited by knocking down the METTL3 (Fig. 2E) and overexpressing the FTO, a $\mathrm{m}^{6} \mathrm{~A}$ demethylases (Fig. $2 \mathrm{~F}$ ). These results provided evidence that HSPA7 harboured high $\mathrm{m}^{6} \mathrm{~A}$ modification level, and thus could be regulated in an $\mathrm{m}^{6} \mathrm{~A}$ dependent manner.

To explore the association between expression of HSPA7 and clinical characteristics, we first compared the expression in the TCGA GBM cohorts stratified by molecular subtypes, IDH1 status, G-CIMP status, MGMT promoter status, age and gender, separately. As shown in Fig. 2G, the HSPA7 expression in the proneural subtype were signaficantly lower than those in the classical and mesenchymal subtypes, and the expression in the mesenchymal samples was the highest. The expression was lower in IDH mutant samples than those with wild-type IDH1. For G-CIMP status, the expression was lower in patients with GCIMP than those without G-CIMP. And there were no obvious changes in the characteristics of MGMT promoter methylation, age and gender. Furthermore, univariate cox regression analysis of overall survival of GBM samples within TCGA showed that high HSPA7 expression (HR:1.511, $\mathrm{P}=0.056$ ) was the independent risk factors associated with prognosis of GBM. And high HSPA7 expression (HR:1.418, $p=$ 0.034) still remained statistically significance in GBM samples, after taking into account age, gender, IDH status, MGMT promoter methylation status and G-CIMP status in subsequent multivariate cox regression 
analysis (Fig. 2H). These results indicated that the pseudogene HSPA7 is a novel prognosis biomarker and indicate therapy outcome for GBM patients.

\section{HSPA7 is correlated with immunophenotypes and TME landscapes.}

To further gain insight into the exact role of HSPA7 in determining immunophenotype, we analyzed 29 immune-associated gene sets, representing diverse immune cell types, functions and pathways (see Methods). As shown in the heatmap (Fig. 3A), we found that HSPA7 high expression group had significantly greater TME cell infiltration characterization, higher immunescore, stromalscore and lower tumorpurity, confirming that HSPA7 could indeed regulate immune cell infiltration and immune related genes expression. And we then explored the specific difference of 16 immune cells between high and low HSPA7 expression patients. We found tumors with high expression of HSPA7 presented significantly increased infiltration in immunosuppression populations, such as macrophages, neutrophils and Tregs (regulatory T cells), compared to patients with low expression; While, some immunity activating cells also enriched, including activated DCs (aDCs), immature DCs (iDCs), plasmacytoid DCs (pDCs) and TILs (Tumor infiltrating lymphocytes) (Fig. S3A), indicating the complexity of TME, in which GBM cells elicited multiple biological behavior changes through direct or indirect interactions with other TME components. Macrophages, neutrophils and Tregs and immune checkpoints, suppressing cytotoxic $T$ lymphocyte (CTL) function, are emerging as critical regulators of cancer immune escape, leading to an immunosuppressive microenvironment that is permissive to tumor outgrowth ${ }^{[32,33]}$. And two recent single-cell mapping of human brain cancer literature ${ }^{[7,8]}$ synergistically reported that abundant cellular component of the brain TME are microglia and monocyte-derived macrophages, having both tumorpromoting and immunosuppressive functions. Moreover, they also found the elevation of neutrophil infiltration and relative frequencies of Tregs in tumor tissues. They also found that both activation and exhaustion of lymphocytes were prevalent. revealing the diversity of, and the dynamic relationships between cellular components, determining the brain TME complexity and multifaceted functions. And the Univariate and multivariate analysis also showed that the macrophage and neutrophils were risk factors in TCGA GBM patients (Table S7). We found that HSPA7 had a significantly positive correlation with the enrichment scores of the three immunosuppressive immune cells and immune checpoints (Fig. 3B). We then explored the specific difference of markers of suppressor function of myeloid lineages (CD33, NOS2, CD163, CD68, SPP1, CD14, CD206), immune inhibitory checkpoints (LAG3, CTLA4, HAVCR2, PDCD1, PDCD1LG1, CD27, TIGIT, CD274 and TNFRSF9) and major neutrophil-recruiting chemokines and their receptors (ITGA3, CD177, MET and CXCL8) between high and low HSPA7 expression patients. The results showed that tumors with high expression of HSPA7 presented significantly increased expression compared to patients with low expression (Fig. 3C-E), and there were also positive correlations between HSPA7 and these molecules (Fig. S4A-C), indicating that HSPA7 could restrain the intra-tumoral antitumor immune response. 
To further understand the exact role of HSPA7 in determining the TME profile and immunophenotype, we performed unsupervised consensus clustering based on the TME cell populations and immune related function genesets, developing a TME pattern with two clusters, TME immune low phenotype (Immune-L) and high (Immune-H) (Fig. S3B). And compared to the Immune-L group (102 patients), patients in Immune-H (51 patients) experienced the outcome of poorer prognosis (Fig. $3 \mathrm{~F}$ ) and higher HSPA7 expression (Fig. 3G). The PN (proneural), CL (classical) and MES (mesenchymal) subtypes of GBM have been most consistently described in the literature, with PN relating to a more favorable outcome and MES relating to poorer survival ${ }^{[23]}$. And the association between MES gene expression signature, characterized by NF1 mutant, with reduced tumor purity, elevated invasion, migration capacity and infiltration of immunosuppressive cells (macrophages or microglia, mesenchymal stem cells or others), has been identified as a common theme across cancer ${ }^{[33,34]}$. Our results showed that majority of MES GBM samples were high expression of HSPA7, as well as high immune-infiltrating phenotypes (Fig. $3 \mathrm{H}-\mathrm{I})$. Furthermore, we found tumors with high expression of HSPA7 presented significantly increased expression in MES phenotype signature ${ }^{[33]}$, compared to patients with low expression (Fig. S4D). Overall, HSPA7 may function as a robust indicator of immunophenotype, significantly correlated with a poorer immune response in GBM.

\section{HSPA7 is correlated with stromal and carcinogenic activation pathways.}

To explore the biological behaviors among these distinct HSPA7 expression samples, we used GSVA algorithm to estimate pathway enrichment scores for each sample (see Methods). Compared to low HSPA7 expression group, high expression group were more markedly enriched in stromal activation pathways (angiogenesis, epithelial mesenchymal transition, VEGF and TGF beta signaling pathways), carcinogenic signaling pathways (hypoxia, apoptosis, PI3K Akt MTOR signaling, glycolysis, KRAS signaling and some others) and immune responses (IL6 Jak stat3 signaling, playing immunosuppressive effects on $\mathrm{T}$ cell function and mediating ICB resistance in cancers ${ }^{[35]}$, inflammatory response, complement and some others). Whereas, they were less enriched in DNA replication, DNA damage response and mismatch repair (MMR) related functions (Fig. 4A). Previous studies demonstrated that the activation of stroma cells in TME can induce the T-cell suppression and oncogenic signaling pathway via a complicated cellular and biological reconfiguration mechanisms ${ }^{[32,36]}$, attenuating tumor response to PD-L1 blockade ${ }^{[27]}$. As shown in Fig. 4B, stromal activation pathways were positively correlated with carcinogenic signaling pathways and immune suppression pathways, while negatively correlated with DNA damage response and repair pathways in TCGA GBM samples. The further analyses for the activity of stroma-related pathways indicated high HSPA7 expression were significantly associated with higher stromal activation signatures (Fig. 4C), constructed by Mariathasan et al. ${ }^{[27]}$, positively correlated with fibroblasts enrichment (Fig. 4D), calculated by MCP counter ${ }^{[37]}$, and positively correlated with the typical stromal cell activation related pathways (Fig. 4E). Furthermore, TGF- $\beta$ is a pleiotropic cytokine associated with poor prognosis in GBM, playing a pro-tumorigenic role by promoting immunosuppression, 
angiogenesis, metastasis, mesenchymal transition, fibroblast activation ${ }^{[27,38-40]}$ and has been proved to promote the progression of GBM in an autocrine signaling loop ${ }^{[41]}$. In our data, compared to low HSPA7 expression group, TGF- $\beta$ ligand (TGFB1), and a TGF- $\beta$ receptor (TGFBR2), two key regulators in stromal activation and EMT pathways ${ }^{[27]}$, and other genes encoding ECM and matricellular proteins (COLA4A1, COLA4A2, ECM1 and FN1) ${ }^{[8]}$, all showed increased expression in high HSPA7 group (Fig. 4F). Previous studies report that ECM can be shaped by monocyte differentiated macrophages via over-expression of the cathepsin proteases CTSB and CTSW[8], which also showed high expression in HSPA7 high group (Fig. 4F). These results suggested that immune cells and stromal cells in the tumor microenvironment can work together to regulate the immunosuppressive microenvironment synergistically, thereby promoting tumor escape.

Then, we explored the genes, positively correlated with HSPA7 (pearson $r \geq 0.3, p \leq 0.05$, Table S8), enriched pathways via metascape database (see Methods). And, they were significantly enriched in immune response, including cytokine-mediated signaling pathway, leukocyte migration, differentiation and some other immune related pathways. Additionally, they can also enriched in the pathways involving the stromal activation, such as extracellular structure organization and response to wounding. These enriched terms interacted with each other to form a protein-protein interaction network (Fig. 5A). Further, enrichment analysis in PaGenBase ${ }^{[42]}$ showed that the genes were almost specifically expressed in spleen, blood, bone marrow and some other peripheral immune cell aggregation tissues (Fig. 5B), suggesting that HSPA7 could indeed promote the infiltration of immune cells into tumor tissues. And the enrichment analysis in TRRUST ${ }^{[43]}$, a TF-target interaction database, showed that the genes were substantially transcriptionally regulated by NFKB1 and RELA, two important transcription factors involving in the NF-KB signalling pathways (Fig. 5C). Further GSEA analysis,using GO and KEGG database, also showed that HSPA7 positively correlated with immune response and extracellular structure organization (Fig. 5D). This demonstrated again that the HSPA7 was of great significance in shaping different TME landscapes.

\section{The functions of HSPA7 was verified in two CGGA cohorts}

To further validate the function of HSAP7 in GBM, we explored the expression pattern in two CGGA RNAseq cohorts. We found that the expression of HSAP7 was highest in GBM (WHO IV), among the three different WHO grade glioma specimens (Fig. S5A, Fig. S6A). Kaplan-Meier survival curve analyses showed that GBM patients with high HSPA7 expression had poor survival outcome in both cohorts (Fig. S5B, Fig. S6B). Further GSVA enrichment analysis also showed that HSPA7 expression correlated with immunophenotypes, stromal activation and oncogenic pathways in GBM samples (Fig. S5C, Fig. S6C). These results confirmed that HSPA7 could be an independent risk prognosis biomarker for GBM patients. Moreover, it can regulate the TME immune response and stromal activation, promoting in the malignant progression of GBM tumors. 


\section{WTAP potentially regulate recruitment of the $\mathrm{m}^{6} \mathrm{~A}$ methyltransferase complex to HSAP7 directly}

To explore the potential molecular mechanism in regulating HSPA7 RNA metabolism, we first screen HSPA7-interacting proteins via NCBI database (Table S9). We verified WTAP ${ }^{[44]}$, a regulatory subunit of the $\mathrm{m}^{6} \mathrm{~A}$ methyltransferase, required for their localization into nuclear speckles, and enhancing the RNAbinding capability of METTL3 to target $\mathrm{RNA}^{[31,45]}$, as putative HSPA7-binding regulator. To better map the association between WTAP and HSPA7, we explored the expression pattern in different grade of glioma in TCGA, CGGA, Gravendeel and Rembrandt datasets. As shown in Fig. S7A, the expression of WTAP is highest in WHO IV (GBM) group, among the three WHO grades glioma and normal brain samples. Further, the overexpression of WTAP correlated with the poor overall survival of GBM patients in all four datasets (Fig. S7B). What's more, the expression of WTAP was postively correlated with the expression of HSPA7 in TCGA GBM dataset (calculated by GEPIA database) and two CGGA GBM datasets (Fig. S7C). Further GSVA enrichment analysis showed that compared to WTAP low expression group, WTAP high group also showed higher TME immune cells infiltration, stromal activation and carcinogenic pathways activation, with higher immunescore, stromalscore and lower tumor purity in TCGA GBM cohort (Fig. S7D), CGGA GBM cohort 1 (Fig. S8A) and CGGA GBM cohort 2 (Fig. S8B), same as HSPA7. From above, we could speculate that $\mathrm{m}^{6} \mathrm{~A}$ methylation modification HSPA7 was regulated by WTAP directly, recruiting the $\mathrm{m}^{6} \mathrm{~A}$ methyltransferase complex. The conclusion and functional mechanisms need to be further demonstrated in the future work.

The following pathway and process enrichment analysis of HSPA7-interacting proteins showed that they can invovled in many biological behaviors, such as mitotic cell cycle phase transition, Neddylation, proteasome-mediated ubiquitin-dependent protein catabolic, HIF1 a pathway and some others (Fig. S9A, Table S9). Then, we further expolred the enriched terms across the RBPs (RNA binding proteins), detected by CLIP-seq data dopoisted in stabase database. We found that they were mainly enriched in mRNA processing, nucleic acid transport, RNA catabolic process and so on (Fig. S9B, Table S10). And we can see component annotation in NCBI database that HSPA7 can be expressed in various regions of the cell (cytoplasm, nucleus, plasma membrane and nucleus), indicating that it may be involved in multiple cellular processes, and the exact mechenism of HSPA7 regulating the GBM TME need us spend a lot of effort and experimentation to clarify in the future.

\section{HSPA7 holds promise in predicting therapeutic response to PD-L1 blockade}

Previous studies indicated that GBM patients is resistant to immune checkpoint inhibitors (ICls), due to its low mutation rate, PTEN-deficient immunosuppressive microenvironment, infiltration by myeloid-derived suppressor cells, and activation of tumor stromal cells ${ }^{[46,47]}$. Then, we analyzed the distribution differences of somatic mutation between low and high HSPA7 expression in TCGA-GBM cohort using 
maftools package, and we found that the mutant rate of PTEN was increased signaficantly in HSPA7 high expression group, compared to the low expression (Fig. 6A, Low: 20\%; High: 36\%). Additionally, the NF1 mutant rate was also elevated obviously (Fig. 6A, Low: $8 \%$; High: $11 \%$ ). Chen et al. ${ }^{[48]}$ find that PTEN deficiency in GBM increases macrophage infiltration via upregulating lysyl oxidase (LOX) expression, in turn secreting SPP1 to support GBM survival. And NF1 mutant is a marker of mesentromal GBM subtype, which also showed that high macrophage infiltration and activation. And then we analysised the association between HSPA7 and macrophage assciated genes ( LOX, SPP1, MACRO, the maker of SPP1 macrophages, and two important chemokines, CCL2 and CCR2). We found that HSPA7 had a postive correlation with these genes significantly (Fig. S10). What's more, Robert M Samstein et. ${ }^{[49]}$ found that TMB predicted survival after immunotherapy across multiple cancer types genomic (including GBM), and we downloaded the mutantion data of $82 \mathrm{GBM}$ samples, and then analyzed the distribution differences of somatic mutation between bottom 30 low TMB and top 30 high TMB samples. We found that the mutant rate of PTEN and NF1 increased signaficantly in low TMB samples, compared to the high group, same results as changes regulated by HSPA7 (Fig. S11A, PTEN: Low, 53\%, High: 30\%; NF1: Low, 20\%, High: 7\%). We further explored the association between HSPA7 expression and TMB. As shown in Fig. S11B, HSPA7 was statically negative with TMB. And Touat $M$ et. ${ }^{[50]}$ recently found that mismatch repair (MMR)-deficient gliomas were characterized by a lack of prominent $T$ cell infiltrates, extensive intratumoral heterogeneity, poor patient survival and a low rate of response to PD-1 blockade. In our data, we also found that high HSPA7 expression group showed the reduced MMR associated pathways (Fig. 4A), which nagatively correlated with the immunesupression, stromal activation and oncogene pathways (Fig. 4B). Some other reserches also showed the same results ${ }^{[27,51]}$. Furthermore, HSPA7 is nagetively correlated with MMR genes, such as MLH1, MSH2, MSH6 and PMS2 (Fig. S11C). These results promoted us that HSPA7 maybe a potential marker for ICB thrrapy.

To further investigate the predicapacity of immune checkpoint therapy of the HSPA7 in GBM. However, there was few published immunotherapy datasets received ICB thrapy. Then we used the melanoma cohort that received anti-CTLA4 therapy ${ }^{[23]}$ and urothelial cancer cohorts that received anti-PD-1 therapy (IMvigor210) to test the immunotherapy response of HSPA7 as a complement. We found that patients with low HSPA7 expression exhibited significantly clinical benefits in anti-CTLA4 cohort (Fig. 6B, antiCTLA cohort, HR 1.927(0.8997-4.129)). Further, compared to HSPA7 high expression group, we found the significant therapeutic benefit and clinical response to anti-CTLA-4 immunotherapy in those with low HSPA7 group (Fig. 6C-D). Same result were also found in the anti-PD-L1 cohort, Kaplan-Meier curve analysis showed that the bottom 50 low HSPA7 expression patients exhibited markedly prolonged survival, compared to those in the top 50 HSPA7 expression group (Fig. 6E, HR 1.670 (1.022-2.792)). Also, there was a significant better therapeutic and clinical response to anti-PD-L1 immunotherapy in HSPA7 low expression group, compared to HSPA7 high group (Fig. 6F-G).

Further GSVA enrichment analysis showed that compared to bottom low HSPA7 expression samples, HSPA7 high group also showed higher TME immune cells infiltration, with higher immunescore, stromalscore and lower tumor purity (Fig. S12A), and higher stromal activation, higher carcinogenic 
pathways activation, as well as lower MRR pathways (Fig. S12B), consistent exactly with those enrienched functions in GBM (Fig. 3A, Fig. 4A, Fig. S6C and Fig. S7C). The PD-L1 expression of immune cells (IC) and tumor cells (TC) was also detected in the IMvigor210 cohort, we then explored the the difference of HSPA7 expression among different expression level groups. As we can see in Fig. 6H-I, patients with higher PD-L1 expression levels, both IC and TC, were observed with higher HSPA7 expression (anova suumary, IC: $p=0.0001$; TC: $p=0.0009$ ), indicating that HSPA7 could upregulated the PD-L1 expression, suppressing the immune activation. Moreover, previous studies indicated that F-box and WD repeat domain containing 7 (FBXW7) is a vital tumor suppressor in varius cancers, controling proteasome-mediated degradation of oncoproteins such as cyclin E, c-Myc, Mcl-1, mTOR, Jun, Notch and so on ${ }^{[52]}$, and its loss-of-function mutation promotes resistance to anti-PD-1 through the downregulation of viral sensing pathways ${ }^{[53]}$. We found that compared to the FBXW7 wildtype group, HSPA7 experssion showed a obvious decrease in mutant group (Fig. 6J). Fibroblast growth factor receptor 1 (FGFR1) is frequently mutanted in various tumors, whose inhibitors have shown promising therapeutic value in several preclinical models ${ }^{[54]}$. Palakurthi $S$ et. found that the combination of FGFR inhibition and PD-1 blockade can promote tumor-intrinsic induction of antitumor immunity ${ }^{[55]}$. And we also found that HSPA7 experssion is siganificantly higher in mutant group, compared to wildtype group (Fig. 6K). Taken together, our work strongly indicated that HSPA7 expression would contribute to predicting the response to immune checkpiont therapy.

\section{Characterization of HSPA7 across 33 cancer types}

We investigated whether HSPA7 expression was correlated with prognosis in pan-cancer patients. The expression of HSPA7 in tumor tissues and GETx normal brain tissues were calculated via GEPIA database. As shown in Fig. S13A, the expression of HSPA7 were statistically lower in ACC, COAD, DLBC, LAML, LUAD, LUSC, READ, THCA and THYM, while signaficantly higher in GBM, KIRC, KIRP and PAAD, compared to the corresponding normal brain tissues. There are also genetic alteration in many other tumors, although not significant. And the COX survival rates and Kaplan-Meier curve analysis were evaluated using the sangerbox tool. The relationships between HSPA7 expression and prognosis of different tumors were shown in Fig. S14-16. Statically, HSPA7 was a risk factor in most cancers (such as ACC, LDBC, CESC and so on). Moreover, we found that it exhibited relatively favourable factor in several cancer types, such as SKCM, KIRP, SARC and so on. We next evoluted the immunescore and stromalscore across 33 cancers from TCGA database. We found that HSPA7 had significantly postive correlations with the immunescore (Fig. S17) and stromalscore (Fig. S18) among almost all 33 cancer types. Moreover, HSPA7 had significantly postive correlations with immune checkpoints expression (Fig. S19A) and immune response (Fig. S19B) in most cancer types, such as GBM, LGG, OV, LUAD and some others. However, there was no relevance in some cancers, such as SKCM, UVM, TGCT, PAAD and so on, indicating that HSPA7 may functioned in other manner other than regulating TME in these cancers, and the molecular mechanisms of this gene needs to be studied in the specific tumor in the future. Together these data highlight HSPA7 may be a key factor, assisting various cancers acquired various immunophenotype, 
and have potentials to be as a consideration in the development of more-effective immunotherapies for GBM and other immunotherapy-resistant tumors. Because different immune cells may play different roles in different tumours, functions of HSPA7 under specific tumor are necessary to explore in the future.

\section{Discussion}

GBM, as one of the most aggressive cancers in brain tumor type, have no effective and sufficient therapies due to its intratumoral heterogeneity and molecular plasticity up to now. ICB therapy is being actively pursued for GBM as a promising treatment option, but very few patients respond it. Thus, seeking markers regulating the brain TME, prominent players, in ICB therapy of cancers, could reveal promising new targets for therapeutic intervention. LncRNAs and $\mathrm{m}^{6} \mathrm{~A}$ modification are emerging as indispensable regulators of TME regulation. Nevertheless, the overall TME infiltration characterizations mediated by $\mathrm{m}^{6} \mathrm{~A}$ modified IncRNAs havn't been comprehensively recognized. Therefore, it is worthy to get a comprehensive knowledge in the TME cell infiltration characterizations mediated by $\mathrm{m}^{6} \mathrm{~A}$ regulated IncRNAs.

Here, based on our $\mathrm{m}^{6} \mathrm{~A}$-seq data, we revealed highly distinct $\mathrm{m}^{6} \mathrm{~A}$ methylation modification patterns on IncRNAs between GBM and normal brain tissues. And we identified immune-stromal- ${ }^{6}{ }^{6} \mathrm{~A}$ related HSPA7 as a novel risk prognostic factor for GBM patients, playing a crucial role in immunophenotype determination, stromal activation and carcinogenic pathways activation, having a robust predictive capacity of ICB immunotherapeutic response. Further, in this study, WTAP, a $\mathrm{m}^{6} \mathrm{~A}$ modification methyltransferase, was identified as a potential regulator of HSAP7 expression. Further analysis showed that WTAP could also regulate the immunephonetype, sromla activation related pathways, same as HSPA7 (Fig. S7-S8). This demonstrated again that the $\mathrm{m}^{6} \mathrm{~A}$ modification was of great important in shaping different TME landscapes. A great deal of literatures have been reported that TME stromal cells activation can restrict the immune infiltration or facilitate the immunesuppressive response in $\mathrm{TME}$, mediating the therapeutic resistance to ICB therapy ${ }^{[27,56]}$. Our data revealed that HSPA7 had a markedly negative correlation tumor mutation burden, a marker of ICB therapy, in TCGA GBM cohorts (Fig. S11B). And a study found that PTEN mutations associated with immunosuppressive expression signatures in non-responders to anti-PD-1 immunotherapy of GBM $^{[2]}$, Chen et al. ${ }^{[48]}$ find that PTEN deficiency in GBM increases SPP ${ }^{+}$macrophage infiltration, which could interract with fibroblasts and vascular endothelial cells, inducing angiogenesis, EMT and some other stormal activation related pathways in colon cancer, whose marker was the pattern recognition receptor MACRO ${ }^{[57]}$, via a YAP1-LOX- $\beta 1$ integrin-PYK2 axis. Intriguingly, antibody-mediated MARCO inhibits cancer progression and metastasis, enhancing ICB efficacy ${ }^{[58]}$, suggestting that SPP $1^{+}$macrophage play a nonnegligible role in the immunerepressive response and immunotherapy resistance. We found that HSPA7 is positively correlated with the expression of LOX, SPP1, MARCO, CCL2 and CCR2, two key chemokines for monocyte infiltration ((Fig. S10). Futhermore, we found HSPA7 could interact with YAP1 ${ }^{[59]}$, which could secrete CCL2/CSF1 to recruit the monocyte ${ }^{[60]}$, promoting us that HSPA7 may be a potential target, synergistically regulating the 
SPP1 ${ }^{+}$macrophages, which then induce the TME stromal activation. What's more, we found that HSPA7 and YAP1 also could be found in GBM extracellular exosome ${ }^{[61]}$, a foundmental regulator of TME cell. And numerous research reports that YAP1 can be as a hub of the network of signals exchanged within the tumour microenvironment ${ }^{[62]}$, regulating the immune response and stromal activation. Of course, the specific mechanism of HSPA7 needs to be proved by a large number of experiments in the following work. We also confirmed the predictive value of the HSPA7 in melanoma cohort that received anti-CTLA4 therapy and urothelial cancer cohorts that received anti-PD-1 therapy (IMvigor210 cohort). And a statistically difference of HSPA7 expression existed between non-respondersand responders. Mismatchrepair (MMR) deficiency recently are emerging as an beneficial indicator of response to PD-1 blockade in patients with cancer ${ }^{[63,64]}$, whereas we found that HSPA7 was nagativle correlated with MMR related pathways (Fig. 4A), which nagativle correlated with stromal activation and carcinogenic pathways(Fig. 4B, Fig.S12), this suggest that MMR deficiency was a unfavourable factor for ICB therapy. Some other reserches also showed the same results ${ }^{[27,51]}$. And a recent study found that MMR-defcient gliomas were characterized by a lack of prominent T cell infltration, extensive intratumoral heterogeneity, poor patient survival and a low rate of response to PD-1 blockade by comprehensively analysing the data of 10,294 samples $^{[50]}$, reconfirming the predictive ability of HSPA7 in immunetherapy response. We also explored the function of HSPA7 in variuos cancer types. As shown in Fig. S13-S19, its expression had prognostic significance in most cancers, and it can regulate the expression of immune checkpoint genes and immune response pathways, as well as a positive correlation with the immune score and stromal score in majority tumor types. It is strange that HSAP7 is a risk factor in melanoma cohort that received anti-CTLA4 therapy (Fig. 6B-D), whereas it is a benefit prognostic factor in TCGA SKCM cohort ${ }^{[23]}$ (Fig. S14, Fig. S16), with no obvious correlation with the immune checkpoint expression (Fig. S19A) and immune response (Fig. S19B). The mechanisms that underlie this divergence need to be explored in the future ceaselessly.

In short, via conjoint analysis of our own $\mathrm{m}^{6} \mathrm{~A}$-seq data and public clinical data, we found a highly distinct $\mathrm{m}^{6} \mathrm{~A}$ methylation modification patterns on IncRNAs between GBM and normal brain tissues, and identified that HSPA7 could be used to evaluate the prognosis of GBM patients, as well as their corresponding TME cell infiltration and activation characterization within individual patient, and predict the efficacy of the patients' clinical response to anti-PD-1/PD-L1 immunotherapy. More importantly, this study offer us several novel insights for cancer immunotherapy that targeting $\mathrm{m}^{6} \mathrm{~A}$ regulators for changing the $\mathrm{m}^{6} \mathrm{~A}$ modification patterns of its key immune regulating targets, may reversing the adverse TME cell infiltration characterization. The study contribute to exploit novel drug combination strategies or novel immunotherapeutic agents in the future.

\section{Conclusion}

In conclusion, this work demonstrated that $\mathrm{m}^{6} \mathrm{~A}$ methylation profiling of IncRNAs in GBM is significantle distinct to the normal brain tissues. And the $\mathrm{m}^{6} \mathrm{~A}$ modified IncRNA HSAP7 played a nonnegligible role in 
TME cell infiltration, stromal and carcinogenic activation, and could be a robust predictive biomarker of ICB immunotherapy for GBM patients. This work contribute to our understanding of the $\mathrm{m}^{6} \mathrm{~A}$ modification in the TME regulation and to exploit more effective immunotherapy strategies.

\section{Abbreviations}

GBM: Glioblastoma; ICB: Immune checkpoint blockade; $\mathrm{m}^{6} \mathrm{~A}$ : $\mathrm{N}^{6}$-Methyladenosine; $\mathrm{M}^{6} \mathrm{~A}$-sequencing: $\mathrm{m}^{6} \mathrm{~A}$ seq; HSPA7: Heat shock protein family A (Hsp70) member 7; ssGSEA: Single-sample gene-set enrichment analysis; FC: Foldchange; TME: microenvironment; TAMs: Tumor-associated macrophages; Immune-H: High immune cell infiltration group; Immune-L: Low immune cell infiltration group; HR: Hazard radio; IDH: Isocitrate dehydrogenase; G-CIMP: CpG island methylator phenotype; MGMT: 06-methylguanineDNA methyltransferase; TCGA: The Cancer Genome Atlas; CGGA: Chinese Glioma Genome Atlas; PN: Proneural; CL: Classical; MES Mesenchymal;

\section{Declarations}

\section{Acknowledgements}

We thank the surgeons and patients who participated in these studies; the Novogene Co. Ltd and Tianjin Novogene Bioinformatics Technology Co., Ltd for $\mathrm{m}^{6} \mathrm{~A}$ technical development and support.

\section{Author's contributions}

GL and HX supervised the project. RRZ designed the research and executed all the results; BYL verified the $\mathrm{m}^{6} \mathrm{~A}$ modification of HSPA7; ZH provided the statistical analysis of $\mathrm{R}$ language code; $\mathrm{SJZ}, \mathrm{QDG}, \mathrm{PZ}$, WQ, SBW and ZHC were responsible for clinical sample collection and the following sample delivery. ZWP, $X G, Y H Q, X L$ and SLZ help to revised the manuscript. All authors read and approved the final manuscript.

\section{Funding}

This work was supported by grants from the National Natural Science Foundation of China (Nos. $81874083 ; 81571284 ; 81702468 ; 81802966 ; 81902540)$, Natural Science Foundation of Shandong Province of China (No. ZR2019BH057), Key clinical Research project of Clinical Research Center of Shandong University (2020SDUCRCA011) and Taishan Scholars of Shandong Province of China (No. ts201511093).

\section{Availability of data and materials}


All data used in this work can be acquired from TCGA database (http://cancergenome.nih.gov/), CGGA database (http://www.cgga.org.cn/) and Supplementary Material. IMvigor210 cohort (http://researchpub.Gene.com/imvigor210corebiologies), cBioPortal database (http://www.cbioportal.org/), and the $\mathrm{m}^{6} \mathrm{~A}$ seq data discussed in this paper have been deposited in PRJNA661159. And the processed data is available from the corresponding author on reasonable request.

\section{Ethics approval and consent to participate}

The research protocol was reviewed and approved by the Ethical Committee on Scientific Research of Shandong University Qilu Hospital (approval number: KYLL-2018-324), and written informed consent was obtained from each patient included in the study; The patient data acquired from the publicly available datasets, whose informed consent of patients were complete.

\section{Consent for publication}

Not applicable.

\section{Competing interests}

The authors declare that they have no competing interests.

\section{Author details}

${ }^{1}$ Department of Neurosurgery, Qilu Hospital, Cheeloo College of Medicine and Institute of Brain and BrainInspired Science, Shandong University, Jinan 250012, Shandong, China;

${ }^{2}$ Shandong Key Laboratory of Brain Function Remodeling, Jinan 250012, Shandong, China.

\section{References}

1. Jackson C, Lim M: Immunotherapy for Glioblastoma: Playing Chess, Not Checkers. Clinical cancer research : an official journal of the American Association for Cancer Research 2018, 24(17):40594061.

2. Zhao J, Chen A, Gartrell R, Silverman A, Aparicio L, Chu T et al: Immune and genomic correlates of response to anti-PD-1 immunotherapy in glioblastoma. Nature medicine 2019, 25(3):462-469.

3. Daniel P, Sabri S, Chaddad A, Meehan B, Jean-Claude B, Rak J et al: Temozolomide Induced Hypermutation in Glioma: Evolutionary Mechanisms and Therapeutic Opportunities. Frontiers in oncology 2019, 9:41. 
4. Johanns T, Miller C, Dorward I, Tsien C, Chang E, Perry A et al: Immunogenomics of Hypermutated Glioblastoma: A Patient with Germline POLE Deficiency Treated with Checkpoint Blockade Immunotherapy. Cancer discovery 2016, 6(11):1230-1236.

5. Bouffet E, Larouche V, Campbell B, Merico D, de Borja R, Aronson M et al: Immune Checkpoint Inhibition for Hypermutant Glioblastoma Multiforme Resulting From Germline Biallelic Mismatch Repair Deficiency. Journal of clinical oncology : official journal of the American Society of Clinical Oncology 2016, 34(19):2206-2211.

6. Gutmann D, Kettenmann H: Microglia/Brain Macrophages as Central Drivers of Brain Tumor Pathobiology. Neuron 2019, 104(3):442-449.

7. Friebel E, Kapolou K, Unger S, Núñez N, Utz S, Rushing E et al: Single-Cell Mapping of Human Brain Cancer Reveals Tumor-Specific Instruction of Tissue-Invading Leukocytes. Cell 2020, 181(7):16261642.e1620.

8. Klemm F, Maas R, Bowman R, Kornete M, Soukup K, Nassiri S et al: Interrogation of the Microenvironmental Landscape in Brain Tumors Reveals Disease-Specific Alterations of Immune Cells. Cell 2020, 181(7):1643-1660.e1617.

9. Deng X, Su R, Weng H, Huang H, Li Z, Chen J: RNA N-methyladenosine modification in cancers: current status and perspectives. Cell research 2018, 28(5):507-517.

10. Yang Y, Hsu P, Chen Y, Yang Y: Dynamic transcriptomic mA decoration: writers, erasers, readers and functions in RNA metabolism. Cell research 2018, 28(6):616-624.

11. Xu C, Yuan B, He T, Ding B, Li S: Prognostic values of YTHDF1 regulated negatively by mir-3436 in Glioma. Journal of cellular and molecular medicine 2020.

12. Dong Z, Cui H: The Emerging Roles of RNA Modifications in Glioblastoma. Cancers 2020, 12(3).

13. Chai R, Wu F, Wang Q, Zhang S, Zhang K, Liu Y et al: mA RNA methylation regulators contribute to malignant progression and have clinical prognostic impact in gliomas. Aging 2019, 11(4):1204-1225.

14. Visvanathan A, Patil V, Arora A, Hegde A, Arivazhagan A, Santosh V et al: Essential role of METTL3mediated $\mathrm{mA}$ modification in glioma stem-like cells maintenance and radioresistance. Oncogene 2018, 37(4):522-533.

15. Zhang S, Zhao B, Zhou A, Lin K, Zheng S, Lu Z et al: mA Demethylase ALKBH5 Maintains Tumorigenicity of Glioblastoma Stem-like Cells by Sustaining FOXM1 Expression and Cell Proliferation Program. Cancer cell 2017, 31(4):591-606.e596.

16. Zheng Q, Hou J, Zhou Y, Li Z, Cao X: The RNA helicase DDX46 inhibits innate immunity by entrapping mA-demethylated antiviral transcripts in the nucleus. Nature immunology 2017, 18(10):1094-1103.

17. Gao Y, Vasic R, Song Y, Teng R, Liu C, Gbyli R et al: mA Modification Prevents Formation of Endogenous Double-Stranded RNAs and Deleterious Innate Immune Responses during Hematopoietic Development. Immunity 2020, 52(6):1007-1021.e1008.

18. Han D, Liu J, Chen C, Dong L, Liu Y, Chang R et al: Anti-tumour immunity controlled through mRNA mA methylation and YTHDF1 in dendritic cells. Nature 2019, 566(7743):270-274. 
19. Li H, Tong J, Zhu S, Batista P, Duffy E, Zhao J et al: mA mRNA methylation controls T cell homeostasis by targeting the IL-7/STAT5/SOCS pathways. Nature 2017, 548(7667):338-342.

20. Patil D, Chen C, Pickering B, Chow A, Jackson C, Guttman M et al: m(6)A RNA methylation promotes XIST-mediated transcriptional repression. Nature 2016, 537(7620):369-373.

21. Ni W, Yao S, Zhou Y, Liu Y, Huang P, Zhou A et al: Long noncoding RNA GAS5 inhibits progression of colorectal cancer by interacting with and triggering YAP phosphorylation and degradation and is negatively regulated by the mA reader YTHDF3. Molecular cancer 2019, 18(1):143.

22. Huang $\mathrm{H}$, Weng $\mathrm{H}$, Chen J: mA Modification in Coding and Non-coding RNAs: Roles and Therapeutic Implications in Cancer. Cancer cell 2020, 37(3):270-288.

23. Van Allen E, Miao D, Schilling B, Shukla S, Blank C, Zimmer L et al: Genomic correlates of response to CTLA-4 blockade in metastatic melanoma. Science (New York, NY) 2015, 350(6257):207-211.

24. Bindea G, Mlecnik B, Tosolini M, Kirilovsky A, Waldner M, Obenauf A et al: Spatiotemporal dynamics of intratumoral immune cells reveal the immune landscape in human cancer. Immunity 2013, 39(4):782-795.

25. Liberzon A, Birger C, Thorvaldsdóttir H, Ghandi M, Mesirov J, Tamayo P: The Molecular Signatures Database (MSigDB) hallmark gene set collection. Cell systems 2015, 1(6):417-425.

26. Kanehisa M, Sato $Y$, Kawashima M, Furumichi $M$, Tanabe M: KEGG as a reference resource for gene and protein annotation. Nucleic acids research 2016, 44:D457-462.

27. Mariathasan S, Turley S, Nickles D, Castiglioni A, Yuen K, Wang Y et al: TGF $\beta$ attenuates tumour response to PD-L1 blockade by contributing to exclusion of T cells. Nature 2018, 554(7693):544-548.

28. Zeng D, Ye Z, Wu J, Zhou R, Fan X, Wang G et al: Macrophage correlates with immunophenotype and predicts anti-PD-L1 response of urothelial cancer. Theranostics 2020, 10(15):7002-7014.

29. Ma X, Bi E, Lu Y, Su P, Huang C, Liu L et al: Cholesterol Induces CD8 T Cell Exhaustion in the Tumor Microenvironment. Cell metabolism 2019, 30(1):143-156.e145.

30. Hazra A, Gogtay N: Biostatistics Series Module 3: Comparing Groups: Numerical Variables. Indian journal of dermatology 2016, 61(3):251-260.

31. Ping X, Sun B, Wang L, Xiao W, Yang X, Wang W et al: Mammalian WTAP is a regulatory subunit of the RNA N6-methyladenosine methyltransferase. Cell research 2014, 24(2):177-189.

32. Tomaszewski W, Sanchez-Perez L, Gajewski T, Sampson J: Brain Tumor Microenvironment and Host State: Implications for Immunotherapy. Clinical cancer research : an official journal of the American Association for Cancer Research 2019, 25(14):4202-4210.

33. Wang Q, Hu B, Hu X, Kim H, Squatrito M, Scarpace L et al: Tumor Evolution of Glioma-Intrinsic Gene Expression Subtypes Associates with Immunological Changes in the Microenvironment. Cancer cell 2017, 32(1):42-56.e46.

34. Wu A, Wei J, Kong L, Wang Y, Priebe W, Qiao W et al: Glioma cancer stem cells induce immunosuppressive macrophages/microglia. Neuro-oncology 2010, 12(11):1113-1125. 
35. Tsukamoto H, Fujieda K, Miyashita A, Fukushima S, Ikeda T, Kubo Y et al: Combined Blockade of IL6 and PD-1/PD-L1 Signaling Abrogates Mutual Regulation of Their Immunosuppressive Effects in the Tumor Microenvironment. Cancer research 2018, 78(17):5011-5022.

36. Chen D, Mellman I: Elements of cancer immunity and the cancer-immune set point. Nature 2017, 541(7637):321-330.

37. Becht E, Giraldo N, Lacroix L, Buttard B, Elarouci N, Petitprez F et al: Estimating the population abundance of tissue-infiltrating immune and stromal cell populations using gene expression. Genome biology 2016, 17(1):218.

38. Lin R, Zhao L: Mechanistic basis and clinical relevance of the role of transforming growth factor- $\beta$ in cancer. Cancer biology \& medicine 2015, 12(4):385-393.

39. Massagué J: TGFbeta in Cancer. Cell 2008, 134(2):215-230.

40. Flavell R, Sanjabi S, Wrzesinski S, Licona-Limón P: The polarization of immune cells in the tumour environment by TGFbeta. Nature reviews Immunology 2010, 10(8):554-567.

41. Rodón L, Gonzàlez-Juncà A, Inda MM, Sala-Hojman A, Martínez-Sáez E, Seoane J: Active CREB1 promotes a malignant TGF $\beta 2$ autocrine loop in glioblastoma. Cancer discovery 2014, 4(10):12301241.

42. Pan J, Hu S, Shi D, Cai M, Li Y, Zou Q et al: PaGenBase: a pattern gene database for the global and dynamic understanding of gene function. PloS one 2013, 8(12):e80747.

43. Han H, Cho J, Lee S, Yun A, Kim H, Bae D et al: TRRUST v2: an expanded reference database of human and mouse transcriptional regulatory interactions. Nucleic acids research 2018, 46:D380D386.

44. Yue Y, Liu J, Cui X, Cao J, Luo G, Zhang Z et al: VIRMA mediates preferential mA mRNA methylation in $3^{\prime}$ UTR and near stop codon and associates with alternative polyadenylation. Cell discovery 2018, 4:10.

45. Liu J, Yue Y, Han D, Wang X, Fu Y, Zhang L et al: A METTL3-METTL14 complex mediates mammalian nuclear RNA N6-adenosine methylation. Nature chemical biology 2014, 10(2):93-95.

46. Jackson C, Choi J, Lim M: Mechanisms of immunotherapy resistance: lessons from glioblastoma. Nature immunology 2019, 20(9):1100-1109.

47. Lim M, Xia Y, Bettegowda C, Weller M: Current state of immunotherapy for glioblastoma. Nature reviews Clinical oncology 2018, 15(7):422-442.

48. Chen P, Zhao D, Li J, Liang X, Li J, Chang A et al: Symbiotic Macrophage-Glioma Cell Interactions Reveal Synthetic Lethality in PTEN-Null Glioma. Cancer cell 2019, 35(6):868-884.e866.

49. Samstein R, Lee C, Shoushtari A, Hellmann M, Shen R, Janjigian Y et al: Tumor mutational load predicts survival after immunotherapy across multiple cancer types. Nature genetics 2019, 51(2):202-206.

50. Touat M, Li Y, Boynton A, Spurr L, lorgulescu J, Bohrson C et al: Mechanisms and therapeutic implications of hypermutation in gliomas. Nature 2020, 580(7804):517-523. 
51. Zhang B, Wu Q, Li B, Wang D, Wang L, Zhou Y: mA regulator-mediated methylation modification patterns and tumor microenvironment infiltration characterization in gastric cancer. Molecular cancer 2020, 19(1):53.

52. Yeh C, Bellon M, Nicot C: FBXW7: a critical tumor suppressor of human cancers. Molecular cancer 2018, 17(1):115.

53. Gstalder C, Liu D, Miao D, Lutterbach B, DeVine A, Lin C et al: Fbxw7Inactivation of Impairs dsRNA Sensing and Confers Resistance to PD-1 Blockade. Cancer discovery 2020.

54. Peng R, Chen Y, Wei L, Li G, Feng D, Liu S et al: Resistance to FGFR1-targeted therapy leads to autophagy via TAK1/AMPK activation in gastric cancer. Gastric cancer : official journal of the International Gastric Cancer Association and the Japanese Gastric Cancer Association 2020.

55. Palakurthi S, Kuraguchi M, Zacharek S, Zudaire E, Huang W, Bonal D et al: The Combined Effect of FGFR Inhibition and PD-1 Blockade Promotes Tumor-Intrinsic Induction of Antitumor Immunity. Cancer immunology research 2019, 7(9):1457-1471.

56. Afik R, Zigmond E, Vugman M, Klepfish M, Shimshoni E, Pasmanik-Chor M et al: Tumor macrophages are pivotal constructors of tumor collagenous matrix. The Journal of experimental medicine 2016, 213(11):2315-2331.

57. Zhang L, Li Z, Skrzypczynska K, Fang Q, Zhang W, O'Brien S et al: Single-Cell Analyses Inform Mechanisms of Myeloid-Targeted Therapies in Colon Cancer. Cell 2020, 181(2):442-459.e429.

58. Georgoudaki A, Prokopec K, Boura V, Hellqvist E, Sohn S, Östling J et al: Reprogramming TumorAssociated Macrophages by Antibody Targeting Inhibits Cancer Progression and Metastasis. Cell reports 2016, 15(9):2000-2011.

59. Liu Y, Zhang X, Lin J, Chen Y, Qiao Y, Guo S et al: CCT3 acts upstream of YAP and TFCP2 as a potential target and tumour biomarker in liver cancer. Cell death \& disease 2019, 10(9):644.

60. Zhang Y, Zhang H, Zhao B: Hippo Signaling in the Immune System. Trends in biochemical sciences 2018, 43(2):77-80.

61. Choi D, Montermini L, Kim D, Meehan B, Roth F, Rak J: The Impact of Oncogenic EGFRvIll on the Proteome of Extracellular Vesicles Released from Glioblastoma Cells. Molecular \& cellular proteomics : MCP 2018, 17(10):1948-1964.

62. Zanconato F, Cordenonsi M, Piccolo S: YAP and TAZ: a signalling hub of the tumour microenvironment. Nature reviews Cancer 2019, 19(8):454-464.

63. Teo M, Seier K, Ostrovnaya I, Regazzi A, Kania B, Moran M et al: Alterations in DNA Damage Response and Repair Genes as Potential Marker of Clinical Benefit From PD-1/PD-L1 Blockade in Advanced Urothelial Cancers. Journal of clinical oncology : official journal of the American Society of Clinical Oncology 2018, 36(17):1685-1694.

64. Plimack E, Dunbrack R, Brennan T, Andrake M, Zhou Y, Serebriiskii I et al: Defects in DNA Repair Genes Predict Response to Neoadjuvant Cisplatin-based Chemotherapy in Muscle-invasive Bladder Cancer. European urology 2015, 68(6):959-967. 
A

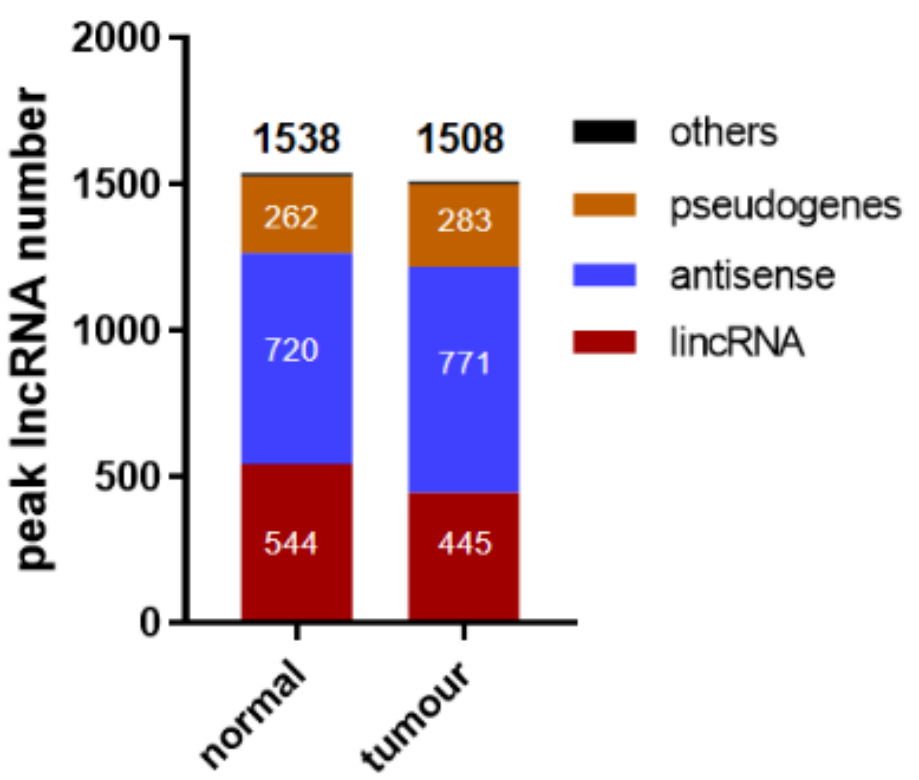

B

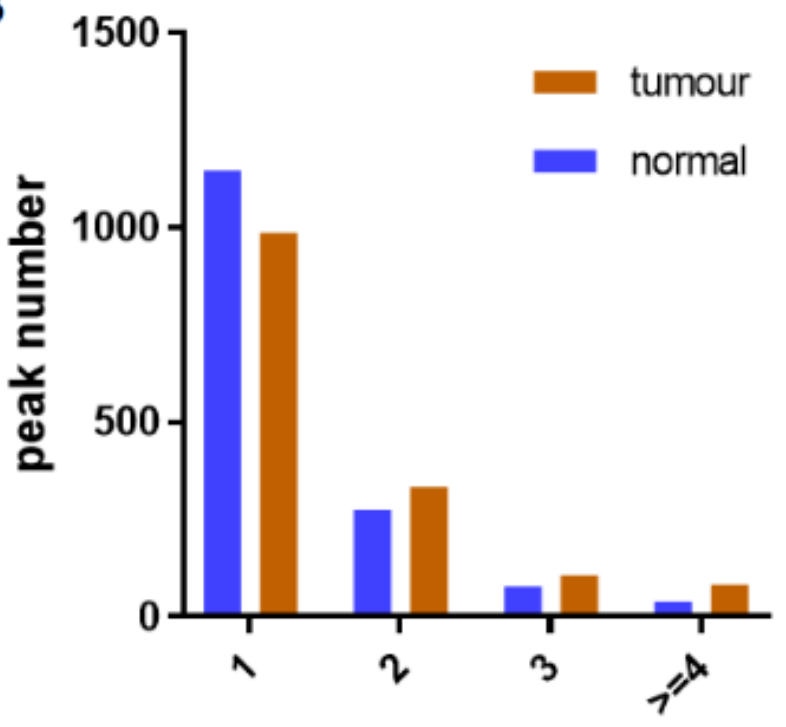

$\mathbf{E}$

pearson correlation: $r=0.495$

C
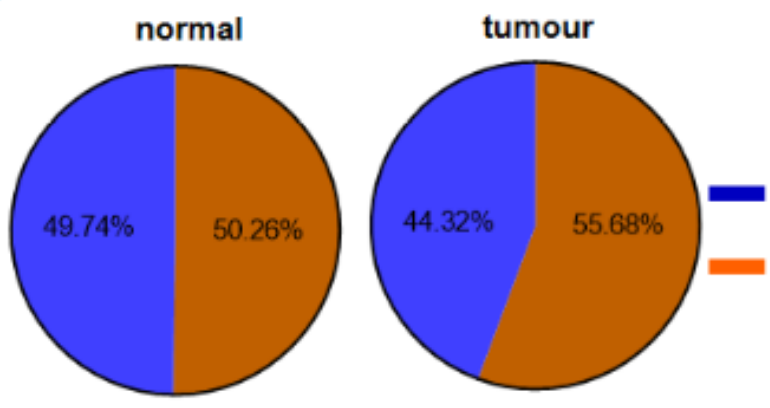

D

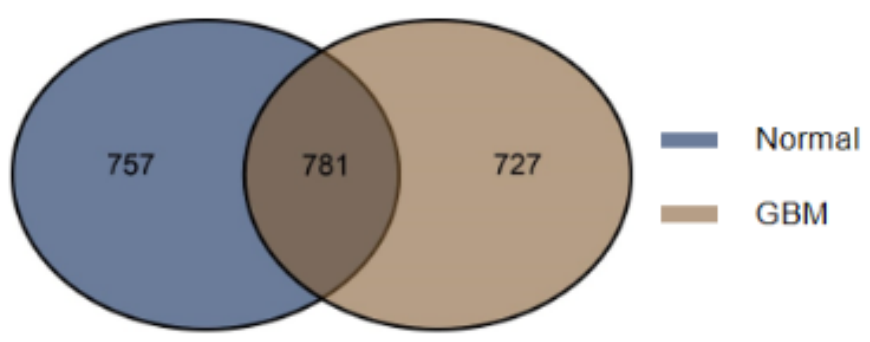
$P<0.0001$

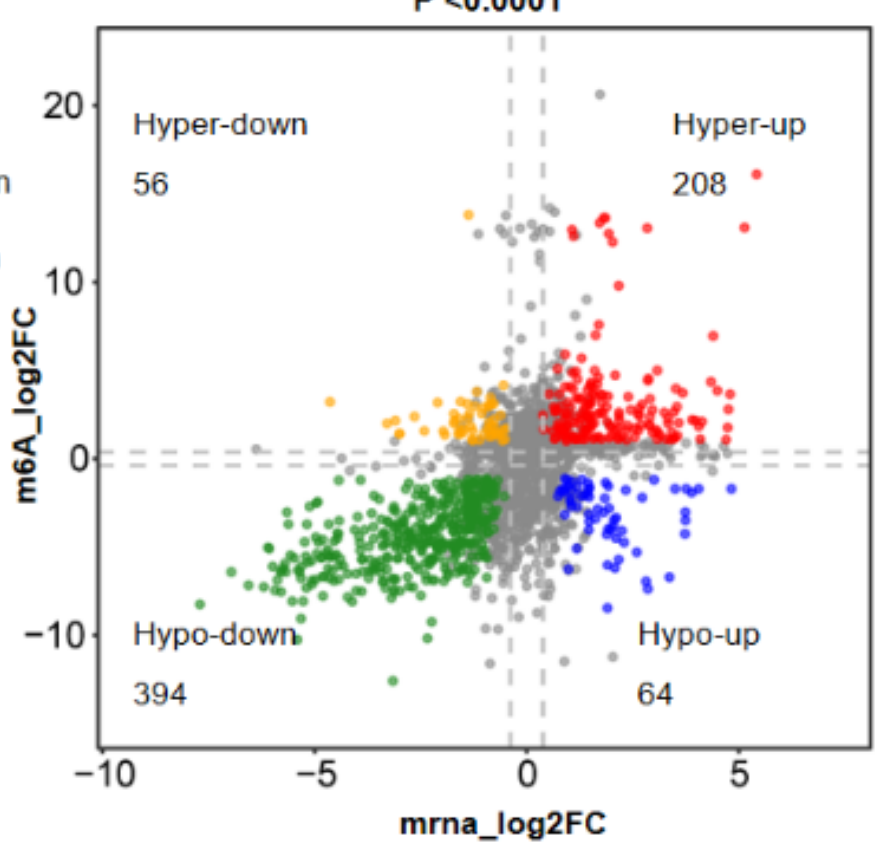

\section{Figure 1}

Overview of m6A methylation within IncRNAs in the GBM and normal brain tissues. (A) The number and gene types of IncRNAs identified in normal brain and GBM tissue samples identified by m6A-seq. (B) The number of m6A-modifed peaks per transcript of IncRNA. (C) Distribution of m6A peaks across IncRNA transcripts. (D) Numbers of common and tissue-specific m6A IncRNAs in the normal and GBM brain tissues. (E) Dot plot of Log2FC (IncRNA expression) against Log2FC (differential m6A methylation) showing a positive correlation between overall m6A methylation and IncRNA expression level (pearson $r=$ 
$0.495 ; p<0.0001)$, and distribution of genes with a significant change in both $\mathrm{m} 6 \mathrm{~A}(\mathrm{FC} \geq 1.2, \mathrm{p} \leq 0.05)$ and corresponding IncRNA expression levels in GBM samples, compared with normal brain tissues (FC $\geq 2$, padj $\leq 0.05)$.

A

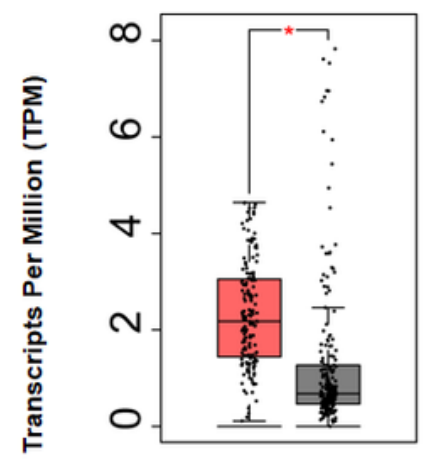

$\operatorname{GBM}(n=163) ; \quad \operatorname{Normal}(n=207)$

C

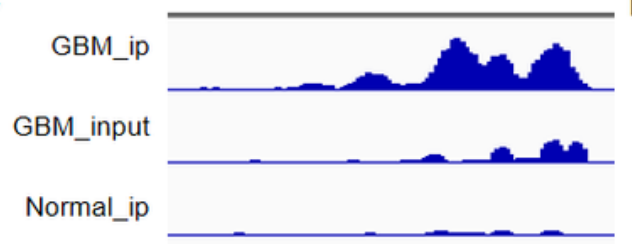

normal_input

HSPA7

G

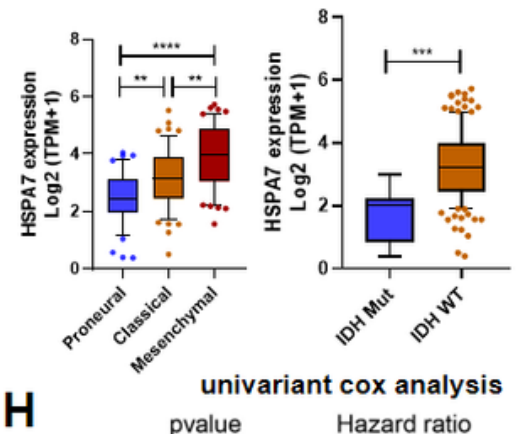

IDH1_status $0.004 \quad 0.185(0.058-0.592)$

MGMT_status $0.006 \quad 0.539(0.347-0.838)$

CIMP_status $0.018 \quad 0.249(0.078-0.792)$

Gender

Age

HSPA7
$0.8631 .039(0.676-1.596)$

$0.008 \quad 1.949(1.189-3.196)$

$0.0561 .511(0.989-2.309)$
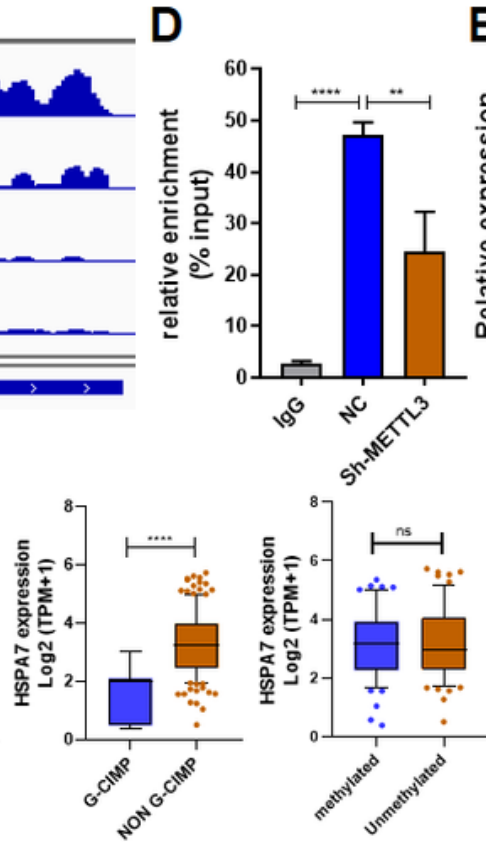

B

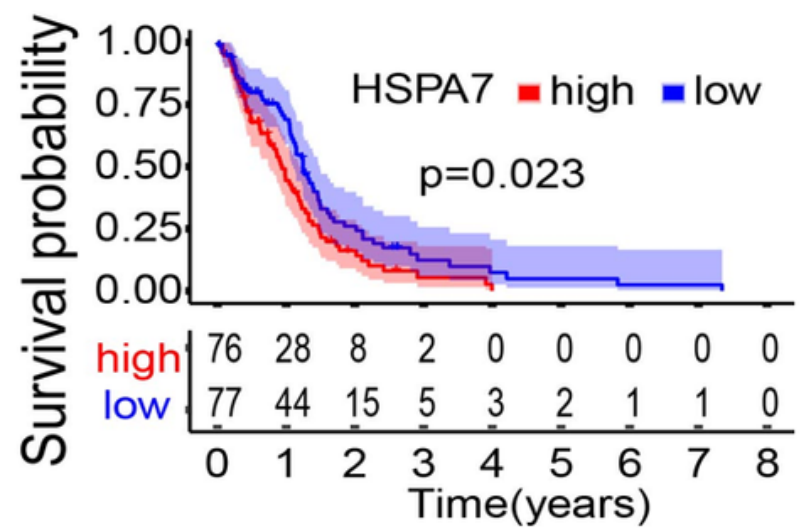

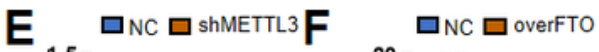
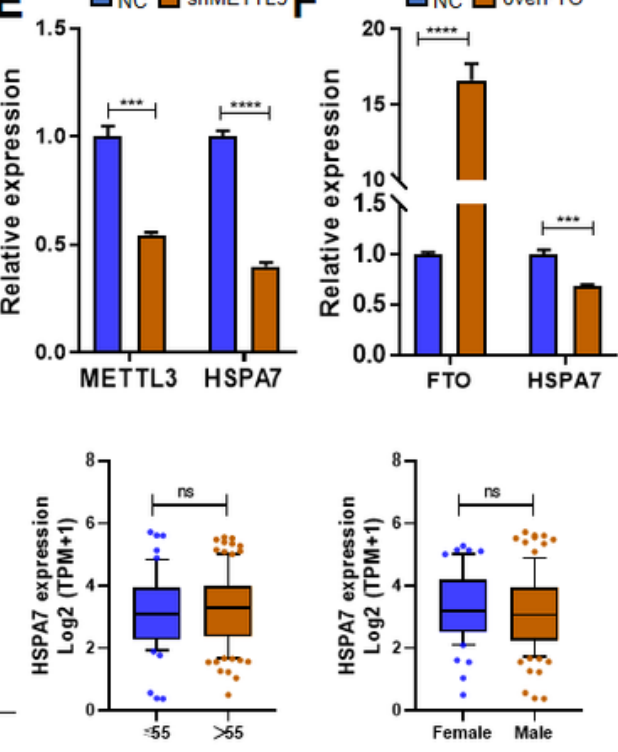

multivariant cox analysis Hazard ratio

pvalue

IDH1_status $0.256 \quad 0.361(0.062-2.096)$

MGMT_status $0.034 \quad 0.594(0.367-0.962)$

CIMP_status $\quad 0.890 \quad 0.889(0.166-4.762)$

Gender

$0.820 \quad 0.945(0.582-1.535)$

$0.0621 .668(0.975-2.854)$

$0.034 \quad 1.418(0.899-2.238)$

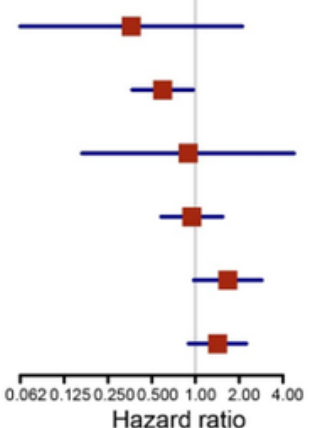

Hazard ratio

Figure 2

Identification of Immune-stromal-m6A-related pseudogene HSPA7 a novel prognostic factor in GBM. (A) GEPIA database show that HSAP7 was over expressed significantly in GBM tissues, compared with GETx 
normal brain tissues. (B) The Kaplan-Meier survival curves show that HSPA7 is a risk prognostic factor in GBM. (C) The gene IGV plots of HSPA7 in the m6A-seq. (D) MeRIP assay showing that HSPA7 was highly enriched by m6A antibody, and the modification can be regulated by m6A methyltransferase, METTL3. Data represent mean \pm S.D. from three independent experiments. QPCR assay showing that the expression of HSPA7 can be regulated by (E) m6A methyltransferase, METTL3, and (F) demethylase FTO. Data represent mean \pm S.D. from three independent experiments. (G) Distribution of the HSPA7 expression in different cohorts stratified by the molecular subtype (Classical, $n=56$; Mesenchymal, $n=$ 51; Proneural, $n=44$; Proneural vs Mesenchymal, $P<0.0001$; Classical vs Mesenchymal, $P=0.0020$; Proneural vs Classical, $P=0.0013$ ), IDH1 status (mutant, $n=8$; wild-type, $n=141 ; P=0.0002$ ), G-CIMP status (G-CIMP, $n=9$; non G-CIMP, $n=142 ; P \otimes 0.0001$ ), MGMT promoter status (methylated, $n=55$; unmethylated, $n=67 ; P=0.7445)$, Age (High, Age > 55, $n=100 ;$ Low, Age $\leq 55, n=53 ; P=0.5114$ ) and Gender (female, $n=54 ;$ male, $n=99 ; P=0.3996)$. $(H)$ Univariate and multivariate Cox regression analysis of HSPA7 and other clinical features in overall survival of GBM samples. 

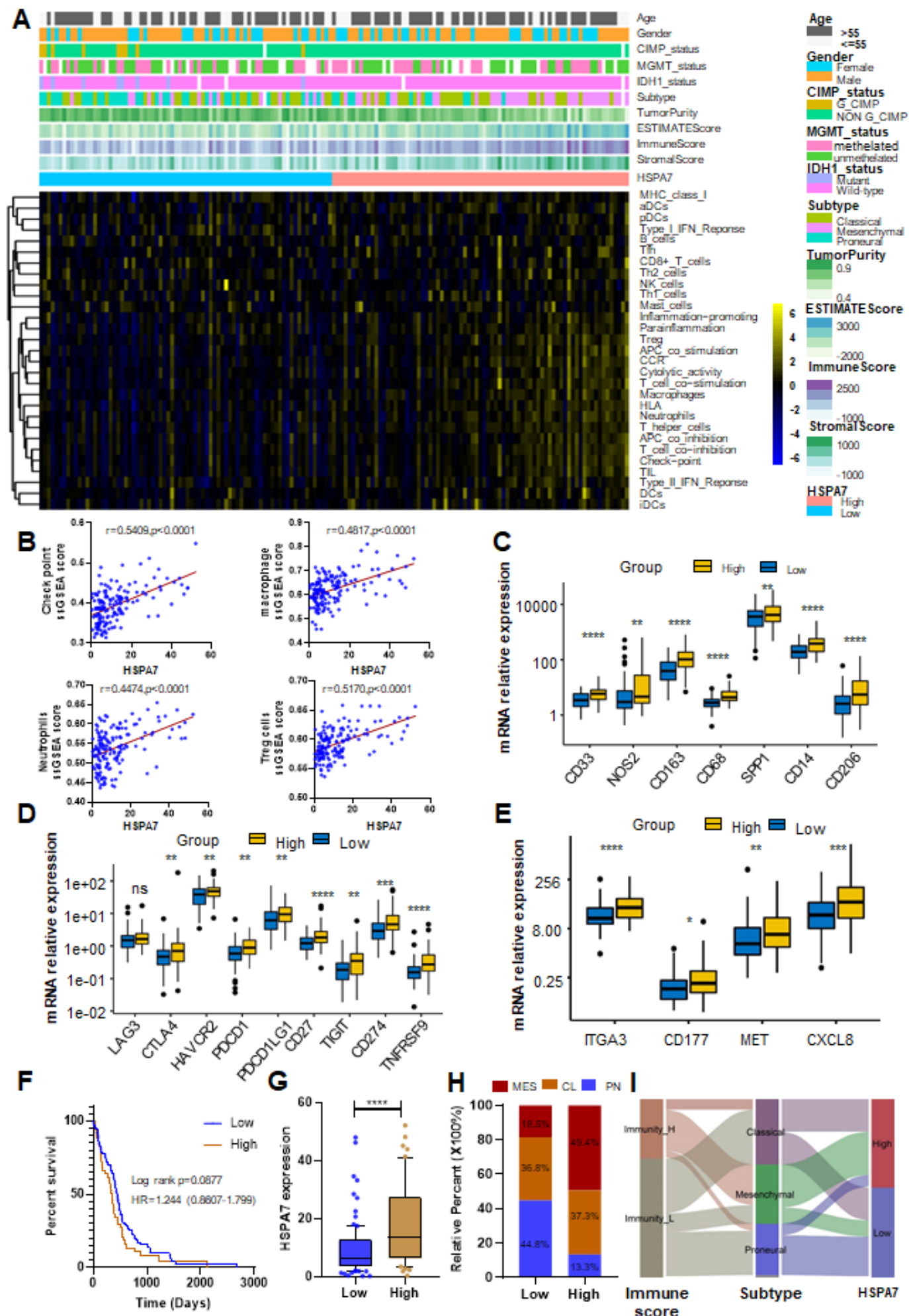

High $曰$ Low
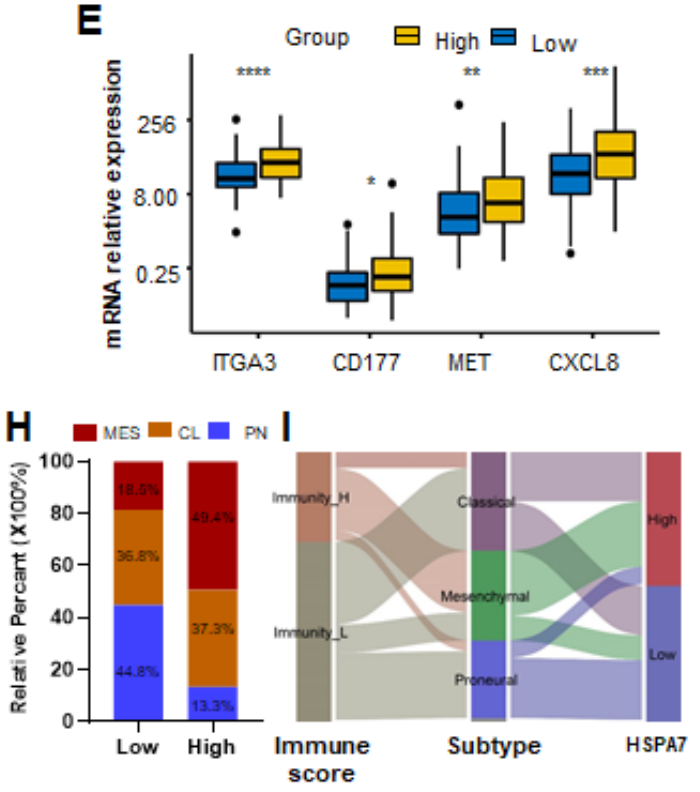

\section{Figure 3}

HSPA7 is correlated with immunophenotypes and TME landscapes. (A) The enrichment scores of immune cell types and immune related function related genesets were calculated via sSGSEA algorithm. The heatmap was used to visualize these immune characteristics between HSAP7 High and Low expression group, and yellow represented high enrichment level, black represented median enrichment level and blue represented low enrichment level. (B) HSPA7 correlated with the immunosuppression 
regulators (check point, macrophage, neutrophil and Treg) positively. High expression of HSPA7 presented significantly increased markers of (C) suppressor function of myeloid lineages, (D) immune inhibitory checkpoints and (E) major neutrophil-recruiting chemokines and their receptors. (F) Compared to the Immune-L group (102 patients), patients in Immune-H (51 patients) experienced the outcome of poorer prognosis. (G) HSPA7 expression was higher in Immune-H group, compared to Immune-L group. $(\mathrm{H})$ The proportion of GBM molecular subtypes in the low and high HSPA7 groups. MES subtype, red; CL subtype, orange; PN subtype, blue. (I) Alluvial diagram showing the changes of immune phenotypes, GBM molecular subtypes and HSPA7 expression. 
A
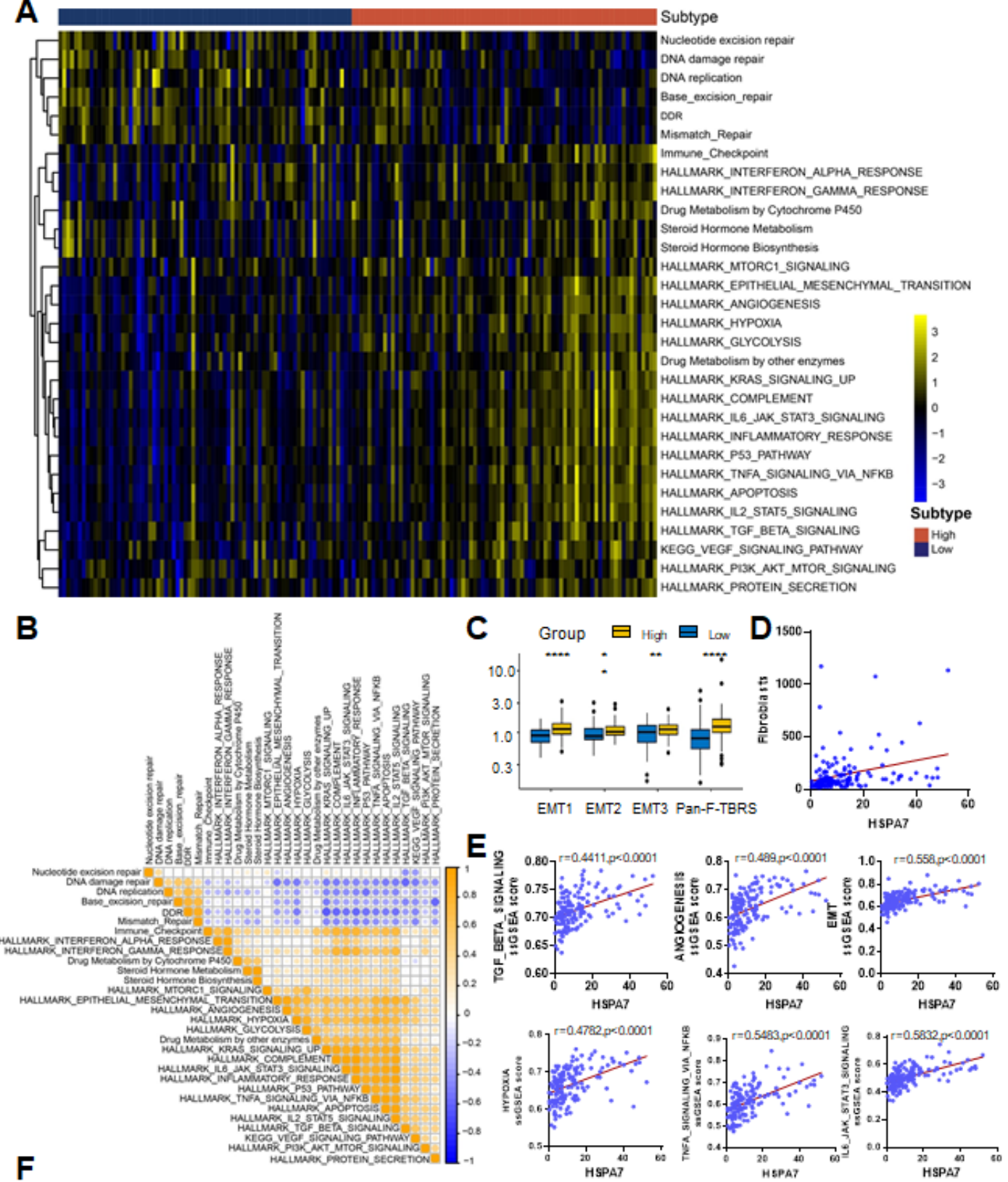

$\mathbf{F}$
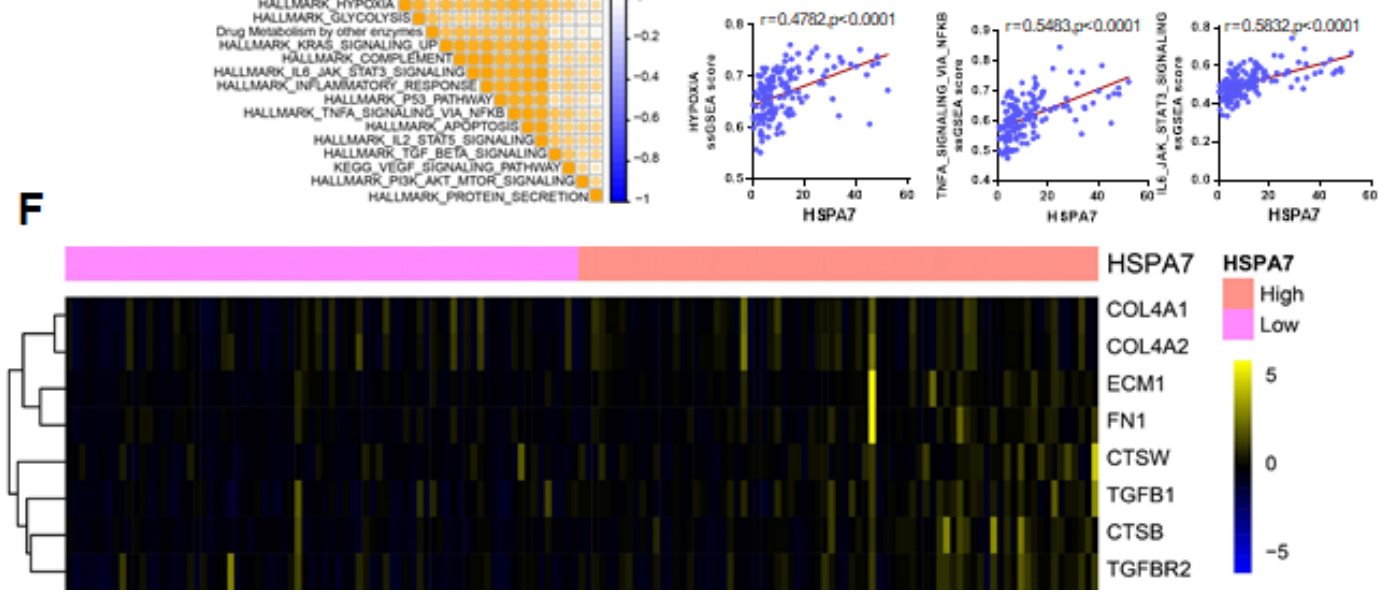

Figure 4

HSPA7 is correlated with stromal and carcinogenic activation pathways. (A) GSVA enrichment analysis showing the activation status of biological pathways in HSPA7 high and low expression groups. The heatmap was used to visualize these biological processes, and yellow represented activated pathways, black represented median activated pathways and blue represented inhibited pathways. (B) Correlations between each known gene signatures in TCGA GBM cohort using pearson analysis. Negative correlation 
was marked with blue and positive correlation with orange. (C) Differences in stroma-activated pathways between high and low HSPA7 expression groups. EMT, epithelial-mesenchymal transition; Pan-FTBRS, pan-fibroblast TGF- $\beta$ response signature. (D) HSPA7 positively correlated with fibroblasts enrichment, calculated by MCP counter. (E) HSPA7 positively correlated with stromal activation related pathways. (F) The heatmap was used to visualize the expression of stromal activation related genes, yellow represented high expression, black represented median expression and blue represented low expression.
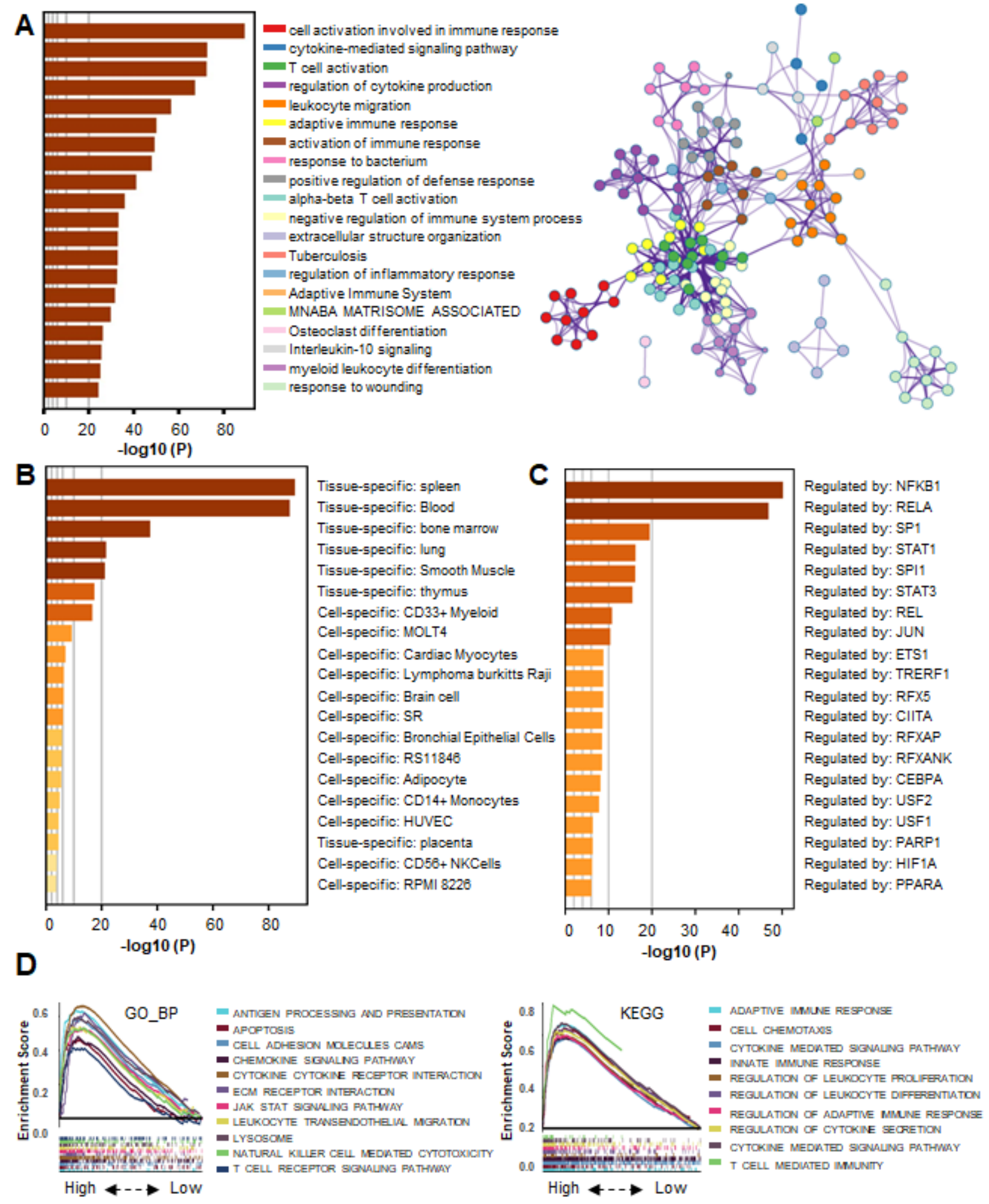

Figure 5 
The genes, postively correlated with HSPA7, were enriched in immune response and stromal related pathways. (A) Bar graph of enriched terms, colored by p-values, and corresponding network of enriched terms. (B) Summary of enrichment analysis in PaGenBase, and (C) summary of enrichment analysis in TRRUST, across HSPA7 positively correlated genes. GSEA analyses displayed key immune regulated pathways enriched in high (up) and low (down) HSPA7 groups, both in GO biological process (left) and KEGG datasets (right), each line is for one pathway.

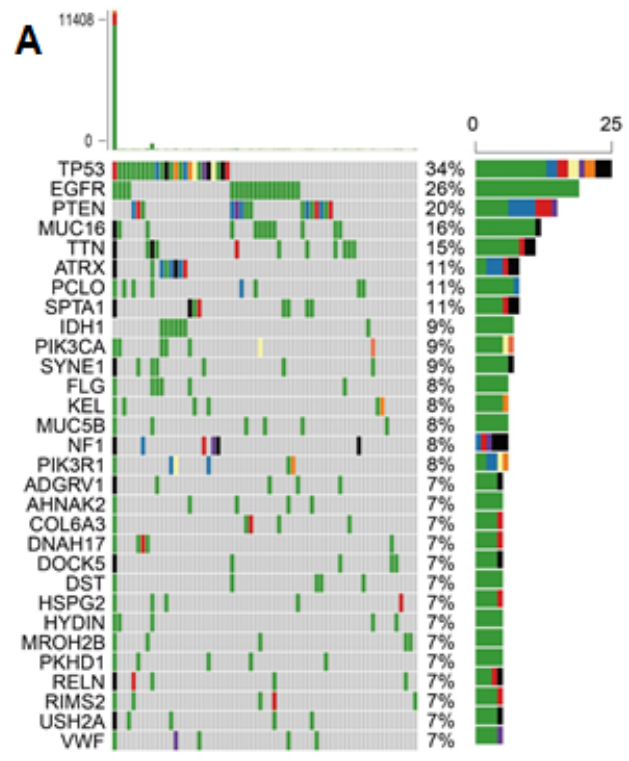

Altered in $65(87.84 \%)$ of 74 samples in Low group.

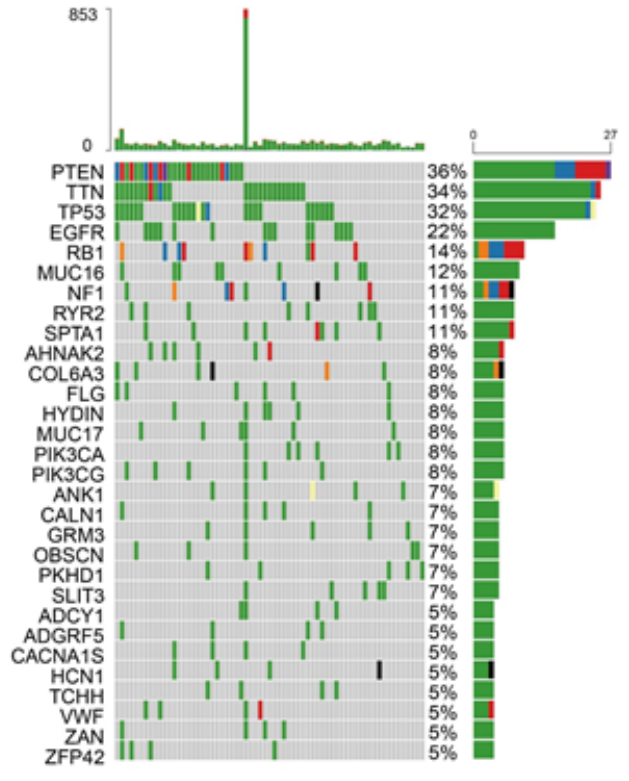

Altered in $65(87.84 \%)$ of 74 samples inHigh group.
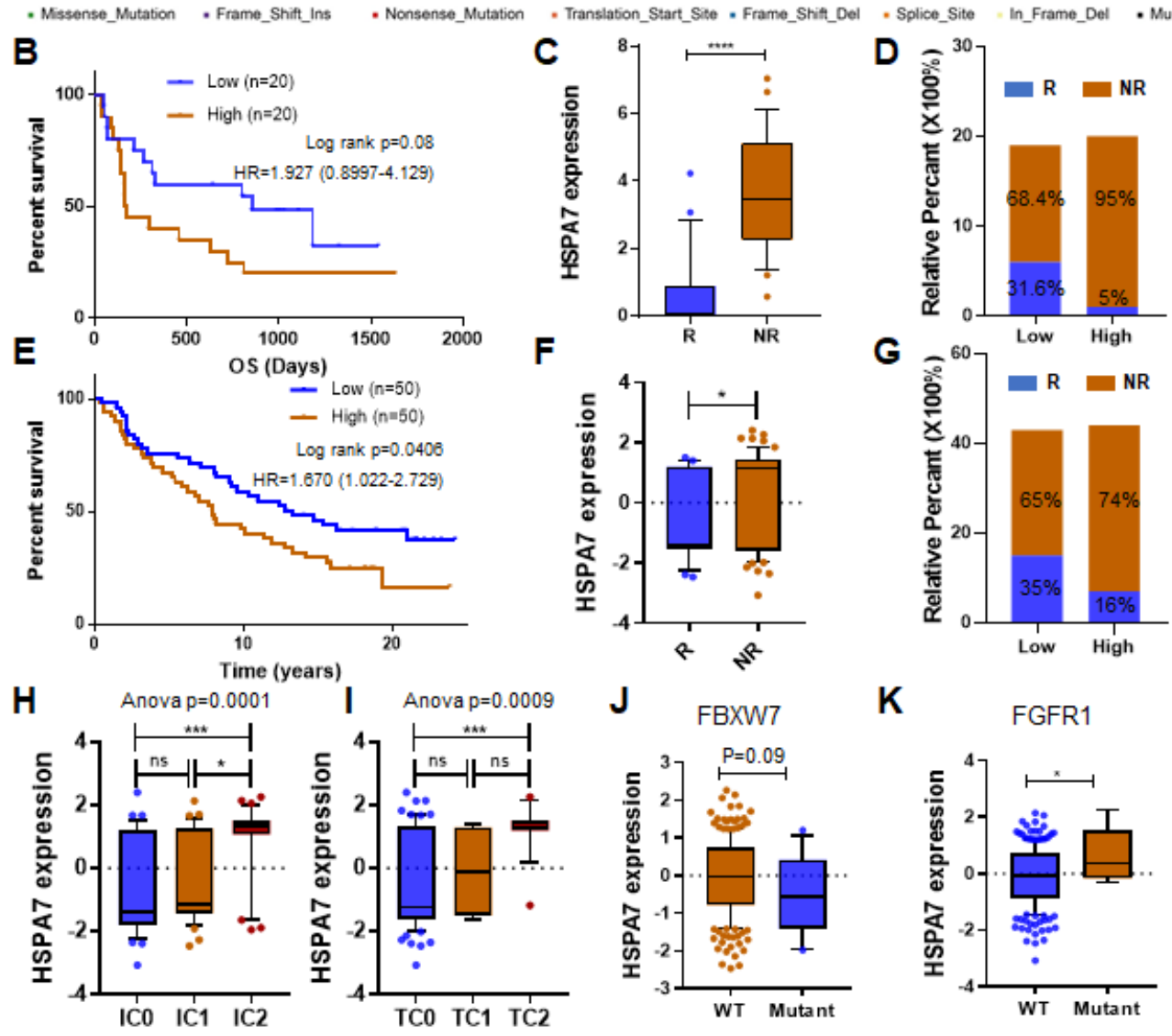

J FBXW7
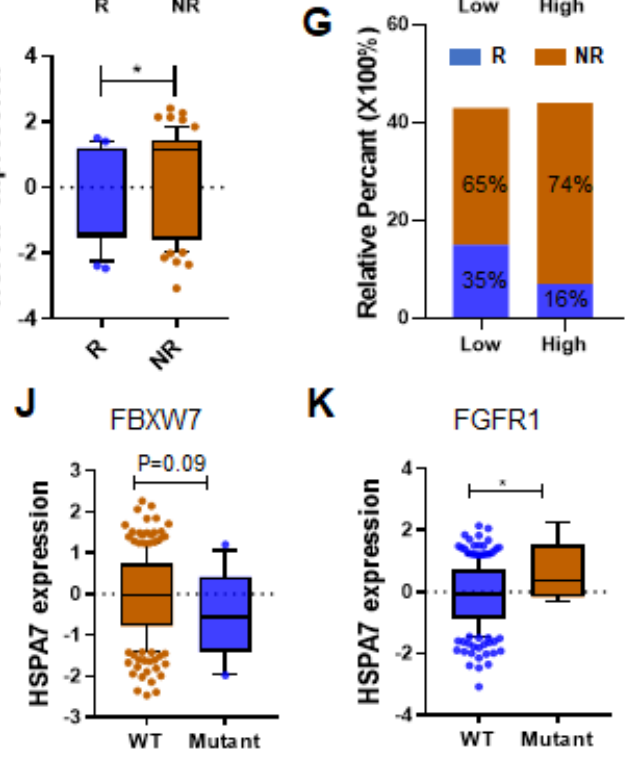

$\mathbf{K}$

FGFR1

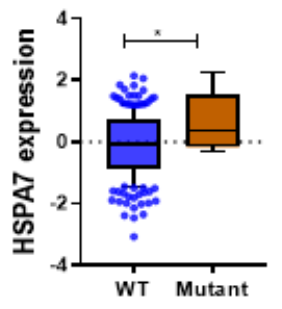

Figure 6 
HSPA7 holds promise in predicting therapeutic response to ICB therapy. (A) The waterfall plot of tumor somatic mutation established by those with high HSPA7 expression group (right) and low group (left). (B) The Kaplan-Meier survival curves showed that HSPA7 is a risk prognostic factor in the melanoma cohort that received anti-CTLA4 therapy. (C) Expression of HSPA7 in distinct anti-CTLA4 clinical response groups, R: response, NR: no response. (D) The proportion of patients with response to CTLA4 blockade immunotherapy in low or high HSPA7 expression groups. (E) The Kaplan-Meier survival curves showed that HSPA7 is a risk prognostic factor in anti-PD-L1 cohort (IMvigor210). (F) Expression of HSPA7 in distinct anti-PD-L1 clinical response groups, R: response, NR: no response. (G) The proportion of patients with response to PD-L1 blockade immunotherapy in low or high HSPA7 expression groups. PD-L1 expression of both IC $(\mathrm{H})$ and TC $(\mathrm{I})$, are associated with HSPA7 expression, with the highest PD-L1 expression level cells showing the highest HSPA7 expression. (Anova, $p=0.0001, p=0.0009$, respectively). HSAP7 expression between (J) FBXW7 wildtype (WT) and mutant status; and (H) FGFR1 wildtype (WT) and mutant status.

\section{Supplementary Files}

This is a list of supplementary files associated with this preprint. Click to download.

- Supplementaryresultsandmethods.docx

- Tables1S7.xlsx

- Tables8.xlsx

- TableS9.xIsx

- Tables10.xlsx 